\title{
Vector Addition System Reachability Problem
}

\author{
A Short Self-Contained Proof
}

\author{
Jérôme Leroux \\ LaBRI, Université de Bordeaux, CNRS \\ leroux@labri.fr
}

\begin{abstract}
The reachability problem for Vector Addition Systems (VASs) is a central problem of net theory. The general problem is known decidable by algorithms exclusively based on the classical KosarajuLambert-Mayr-Sacerdote-Tenney decomposition (KLMTS decomposition). Recently from this decomposition, we deduced that a final configuration is not reachable from an initial one if and only if there exists a Presburger inductive invariant that contains the initial configuration but not the final one. Since we can decide if a Preburger formula denotes an inductive invariant, we deduce from this result that there exist checkable certificates of non-reachability in the Presburger arithmetic. In particular, there exists a simple algorithm for deciding the general VAS reachability problem based on two semi-algorithms. A first one that tries to prove the reachability by enumerating finite sequences of actions and a second one that tries to prove the non-reachability by enumerating Presburger formulas. In this paper we provide the first proof of the VAS reachability problem that is not based on the KLMST decomposition. The proof is based on the notion of production relations inspired from Hauschildt that directly provides the existence of Presburger inductive invariants.
\end{abstract}

Categories and Subject Descriptors D.2.4 [Software Engineering]: Formal methods; D.2.2 [Design Tools and Techniques]: Petri nets

General Terms Theory, Verification

Keywords VAS, Petri, Presburger, Reachability

\section{Introduction}

Vector Addition Systems (VASs) or equivalently Petri Nets are one of the most popular formal methods for the representation and the analysis of parallel processes [EN94]. The reachability problem is central since many computational problems (even outside the parallel processes) reduce to the reachability problem. Sacerdote and Tenney provided in [ST77] a partial proof of decidability of this problem. The proof was completed in 1981 by Mayr [May81] and simplified by Kosaraju [Kos82] from [ST77, May81]. Ten years later [Lam92], Lambert provided a more simplified version based on [Kos82]. This last proof still remains difficult and the upperbound complexity of the corresponding algorithm is just known

Permission to make digital or hard copies of all or part of this work for personal or classroom use is granted without fee provided that copies are not made or distributed for profit or commercial advantage and that copies bear this notice and the full citation on the first page. To copy otherwise, to republish, to post on servers or to redistribute to lists, requires prior specific permission and/or a fee.

POPL'11, January 26-28, 2011, Austin, Texas, USA.

Copyright (C) 2011 ACM 978-1-4503-0490-0/11/01_..\$10.00 non-primitive recursive. Nowadays, the exact complexity of the reachability problem for VASs is still an open-problem. Even an elementary upper-bound complexity is open. In fact, the known general reachability algorithms are exclusively based on the KosarajuLambert-Mayr-Sacerdote-Tenney (KLMST) decomposition.

Recently [Ler09] we proved thanks to the KLMST decomposition that Parikh images of languages accepted by VASs are semipseudo-linear, a class that extends the Presburger sets. An application of this result was provided; we proved that a final configuration is not reachable from an initial one if and only if there exists a forward inductive invariant definable in the Presburger arithmetic that contains the initial configuration but not the final one. Since we can decide if a Presburger formula denotes a forward inductive invariant, we deduce that there exist checkable certificates of nonreachability in the Presburger arithmetic. In particular, there exists a simple algorithm for deciding the general VAS reachability problem based on two semi-algorithms. A first one that tries to prove the reachability by enumerating finite sequences of actions and a second one that tries to prove the non-reachability by enumerating Presburger formulas.

In this paper we provide a new proof of the reachability problem that is not based on the KLMST decomposition. The proof is based on the production relations inspired by Hauschildt [Hau90] and it provides directly that reachability sets are Lambert sets, a class of sets introduced in this paper that extend the class of Presburger sets and contained in the class of semi-pseudo-linear sets. In particular this paper provides a more precise characterization of the reachability sets of VASs.

Outline of the paper: Section 2 provides notations and classical definitions. Section 3 and Section 4 introduce classes of sets used in the sequel : polytope conic sets and vector spaces in the first one and polytope periodic sets, Presburger sets, Lambert sets, and Petri sets in the second one. Section 5 and Section 6 show that it sufficient to prove that the reachability relation of a Vector Addition system is a Petri relation in order to deduce the existence of forward inductive invariants definable in the Presburger arithmetic proving the non-reachability. In Section 7 we introduce the class of Vector Addition Systems and the central notion of production relations. We show in the next Section 8 that these relations are polytope periodic. In Section 9 we prove that the reachability relation of a Vector Addition System is a Petri relation. Finally in Section 10 we combine all the previous results to deduce the decidability of the Vector Addition System reachability problem based on Presburger inductive invariants.

\section{Notations}

We introduce in this section notations and classical definitions used in this paper. 
We denote by $\mathbb{N}, \mathbb{N}_{>0}, \mathbb{Z}, \mathbb{Q}, \mathbb{Q} \geq 0, \mathbb{Q}>0$ the set of natural numbers, positive integers, integers, rational numbers, non negative rational numbers, and positive rational numbers. Vectors and sets of vectors are denoted in bold face. The $i$ th component of a vector $\mathbf{v} \in$ $\mathbb{Q}^{d}$ is denoted by $\mathbf{v}(i)$. We introduce $\|\mathbf{v}\|_{\infty}=\max _{1 \leq i \leq d}|\mathbf{v}(i)|$ where $|\mathbf{v}(i)|$ is the absolute value of $\mathbf{v}(i)$. The total order $\leq$ over $\mathbb{Q}$ is extended component-wise into an order $\leq$ over the set of vectors $\mathbb{Q}^{d}$. The addition function + is also extended component-wise over $\mathbb{Q}^{d}$. Given two sets $\mathbf{V}_{1}, \mathbf{V}_{2} \subseteq \mathbb{Q}^{d}$ we denote by $\mathbf{V}_{1}+\mathbf{V}_{2}$ the set $\left\{\mathbf{v}_{1}+\mathbf{v}_{2} \mid\left(\mathbf{v}_{1}, \mathbf{v}_{2}\right) \in \mathbf{V}_{1} \times \mathbf{V}_{2}\right\}$, and we denote by $\mathbf{V}_{1}-\mathbf{V}_{2}$ the set $\left\{\mathbf{v}_{1}-\mathbf{v}_{2} \mid\left(\mathbf{v}_{1}, \mathbf{v}_{2}\right) \in \mathbf{V}_{1} \times \mathbf{V}_{2}\right\}$. In the same way given $T \subseteq \mathbb{Q}$ and $\mathbf{V} \subseteq \mathbb{Q}^{d}$ we let $T \mathbf{V}=\{t \mathbf{v} \mid(t, \mathbf{v}) \in T \times \mathbf{V}\}$. We also denote by $\mathbf{v}_{1}+\mathbf{V}_{2}$ and $\mathbf{V}_{1}+\mathbf{v}_{2}$ the sets $\left\{\mathbf{v}_{1}\right\}+\mathbf{V}_{2}$ and $\mathbf{V}_{1}+\left\{\mathbf{v}_{2}\right\}$, and we denote by $t \mathbf{V}$ and $T \mathbf{v}$ the sets $\{t\} \mathbf{V}$ and $T\{\mathbf{v}\}$. In the sequel, an empty sum of sets included in $\mathbb{Q}^{d}$ denotes the set reduced to the zero vector $\{\mathbf{0}\}$.

A (binary) relation $R$ over $\mathbb{Q}^{d}$ is a subset $R \subseteq \mathbb{Q}^{d} \times \mathbb{Q}^{d}$. The composition of two relations $R$ and $S$ is the relation denoted by $R \circ S$ and defined as usual by the following equality:

$R \circ S=\bigcup_{\mathbf{y} \in \mathbb{Q}^{d}}\left\{(\mathbf{x}, \mathbf{z}) \in \mathbb{Q}^{d} \times \mathbb{Q}^{d} \mid(\mathbf{x}, \mathbf{y}) \in R \wedge(\mathbf{y}, \mathbf{z}) \in S\right\}$

The reflexive and transitive closure of a relation $R$ is denoted by $R^{*}$. In this paper, notions introduced over the sets are transposed over the relations by identifying $\mathbb{Q}^{d} \times \mathbb{Q}^{d}$ with $\mathbb{Q}^{2 d}$.

An order $\sqsubseteq$ over a set $S$ is said to be well if for every sequence $\left(s_{n}\right)_{n \in \mathbb{N}}$ of elements $s_{n} \in S$ we can extract a sub-sequence that is non-decreasing for $\sqsubseteq$, i.e. there exists a strictly increasing sequence $\left(n_{k}\right)_{k \in \mathbb{N}}$ of natural numbers in $(\mathbb{N}, \leq)$ such that $\left(s_{n_{k}}\right)_{k \in \mathbb{N}}$ is non decreasing for $\sqsubseteq$. A minimal element of an ordered set $(S, \sqsubseteq)$ is an element $s \in S$ such that for every $t \in T$ the relation $t \sqsubseteq s$ implies $s=t$. Given a set $Y \subseteq S$ we denote by $\min _{\sqsubseteq}(Y)$ the set of minimal elements of the ordered set $(Y, \sqsubseteq)$. Let us recall that if $(S, \sqsubseteq)$ is well ordered then $X=\min _{\sqsubseteq}(Y)$ is finite and for every $y \in Y$ there exists $x \in X$ such that $x \sqsubseteq y$.

Let us consider an order $\sqsubseteq$ over a set $S$. We introduce the component-wise extension of $\sqsubseteq$ over the set of vectors $S^{d}$ defined by $\mathbf{s} \sqsubseteq \mathbf{t}$ if $\mathbf{s}(i) \sqsubseteq \mathbf{t}(i)$ for every $i \in\{1, \ldots, d\}$.

Lemma 2.1 (Dickson's Lemma). The ordered set $\left(S^{d}, \sqsubseteq\right)$ is well for every well ordered set $(S, \sqsubseteq)$.

Example 2.2. The set $(\mathbb{N}, \leq)$ is well ordered. Hence $\left(\mathbb{N}^{d}, \leq\right)$ is also well ordered. The set $(\mathbb{Z}, \leq)$ is not well ordered.

\section{Polytope Conic Sets}

In this section we introduce the polytope conic sets and we provide a characterization of this class based on the vector spaces.

A conic set is a set $\mathbf{C} \subseteq \mathbb{Q}^{d}$ such that $\mathbf{0} \in \mathbf{C}, \mathbf{C}+\mathbf{C} \subseteq \mathbf{C}$ and such that $\mathbb{Q}_{\geq 0} \mathbf{C} \subseteq \mathbf{C}$. A conic set $\mathbf{C}$ is said to be finitely generated if there exists a finite sequence $\mathbf{c}_{1}, \ldots, \mathbf{c}_{k}$ of vectors $\mathbf{c}_{j} \in \mathbf{C}$ such that $\mathbf{C}=\mathbb{Q} \geq 0 \mathbf{c}_{1}+\cdots+\mathbb{Q}_{\geq 0} \mathbf{c}_{k}$.

Definition 3.1. A conic set $\mathbf{C}$ is said to be polytope if it is definable in $\mathrm{FO}(\mathbb{Q},+, \leq, 0,1)$.

Example 3.2. The conic set $\mathbf{C}=\left\{\mathbf{c} \in \mathbb{Q}_{\geq 0}^{2} \mid \mathbf{c}(1) \leq \sqrt{2} \mathbf{c}(2)\right\}$ is not polytope.

Example 3.3. The conic set $\mathbf{C}=\{(0,0)\} \cup \mathbb{Q}_{>0}^{2}$ is polytope but it is not finitely generated.

A vector space is a set $\mathbf{V} \subseteq \mathbb{Q}^{d}$ such that $\mathbf{0} \in \mathbf{V}, \mathbf{V}+\mathbf{V} \subseteq \mathbf{V}$ and such that $\mathbb{Q} \mathbf{V} \subseteq \mathbf{V}$. Let $\mathbf{X} \subseteq \mathbb{Q}^{d}$. The following set is a vector space called the vector space generated by $\mathbf{X}$.

$$
\mathbf{V}=\left\{\sum_{j=1}^{k} \lambda_{j} \mathbf{x}_{j} \mid k \in \mathbb{N} \text { and }\left(\lambda_{j}, \mathbf{x}_{j}\right) \in \mathbb{Q} \times \mathbf{X}\right\}
$$

Observe that this vector space is the minimal for the inclusion vector space that contains $\mathbf{X}$. Note that the vector space $\mathbf{V}$ generated by a conic set $\mathbf{C}$ satisfies the equality $\mathbf{V}=\mathbf{C}-\mathbf{C}$. Let us recall that every vector space $\mathbf{V}$ is generated by a finite set $\mathbf{X}$ with at most $d$ vectors. The rank $\operatorname{rank}(\mathbf{V})$ of a vector space $\mathbf{V}$ is the minimal natural number $r \in\{0, \ldots, d\}$ such that there exists a finite set $\mathbf{X}$ with $r$ vectors that generates $\mathbf{V}$. Note that $\operatorname{rank}(\mathbf{V}) \leq \operatorname{rank}(\mathbf{W})$ for every vector spaces $\mathbf{V} \subseteq \mathbf{W}$. Moreover if $\mathbf{V}$ is strictly included in $\mathbf{W}$ then $\operatorname{rank}(\mathbf{V})<\operatorname{rank}(\mathbf{W})$.

The (topological) closure of a set $\mathbf{X} \subseteq \mathbb{Q}^{d}$ is the set $\overline{\mathbf{X}}$ of vectors $\mathbf{r} \in \mathbb{Q}^{d}$ such that for every $\epsilon \in \mathbb{Q}_{>0}$ there exists $\mathbf{x} \in \mathbf{X}$ satisfying $\|\mathbf{r}-\mathbf{x}\|_{\infty}<\epsilon$. A set $\mathbf{X}$ is said to be closed if $\overline{\mathbf{X}}=\mathbf{X}$. Note that $\overline{\mathbf{X}}$ is closed and this set is the minimal for the inclusion closed set that contains $\mathbf{X}$. Let us recall that a vector space $\mathbf{V}$ is closed and the closure of a conic set is a conic set. Since the classical topological interior of a conic set $\mathbf{C}$ is empty when the vector space generated by $\mathbf{C}$ is not equal to $\mathbb{Q}^{d}$ (the conic set is degenerated), we introduce the notion of interior of $\mathbf{C}$ relatively to the vector space $\mathbf{V}=\mathbf{C}-\mathbf{C}$. More precisely, a vector $\mathbf{c} \in \mathbf{C}$ is said to be in the interior of $\mathbf{C}$ if there exists $\epsilon \in \mathbb{Q}>0$ such that $\mathbf{c}+\mathbf{v} \in \mathbf{C}$ for every $\mathbf{v} \in \mathbf{C}-\mathbf{C}$ satisfying $\|\mathbf{v}\|_{\infty}<\epsilon$. We denote by $\operatorname{int}(\mathbf{C})$ the set of interior vectors of $\mathbf{C}$. Let us recall that $\operatorname{int}(\mathbf{C})$ is non empty for every conic set $\mathbf{C}$, and $\overline{\mathbf{C}_{1}}=\overline{\mathbf{C}_{2}}$ if and only if $\operatorname{int}\left(\mathbf{C}_{1}\right)=\operatorname{int}\left(\mathbf{C}_{2}\right)$ for every conic sets $\mathbf{C}_{1}, \mathbf{C}_{2}$.

The following lemma characterizes the finitely generated cones. It proves that finitely generated conic sets are polytope.

Lemma 3.4. Let $\mathbf{V} \subseteq \mathbb{Q}^{d}$ be a vector space. A conic set $\mathbf{C} \subseteq \mathbf{V}$ is finitely generated if and only if there exists a sequence $\left(\mathbf{h}_{j}\right)_{1 \leq j \leq k}$ of vectors $\mathbf{h}_{j} \in \mathbf{V} \backslash\{\mathbf{0}\}$ such that:

$$
\mathbf{C}=\bigcap_{j=1}^{k}\left\{\mathbf{v} \in \mathbf{V} \mid \sum_{i=1}^{d} \mathbf{h}_{j}(i) \mathbf{v}(i) \geq 0\right\}
$$

Moreover in this case the following equality holds if and only if $\mathbf{V}$ is the vector space generated by $\mathbf{C}$ :

$$
\operatorname{int}(\mathbf{C})=\bigcap_{j=1}^{k}\left\{\mathbf{v} \in \mathbf{V} \mid \sum_{i=1}^{d} \mathbf{h}_{j}(i) \mathbf{v}(i)>0\right\}
$$

Proof. This is a classical result of duality [Sch87].

Theorem 3.5. A conic set $\mathbf{C} \subseteq \mathbb{Q}^{d}$ is polytope if and only if the conic set $\overline{\mathbf{C} \cap \mathbf{V}}$ is finitely generated for every vector space $\mathbf{V} \subseteq \mathbb{Q}^{d}$.

Proof. Let us first consider a polytope conic set $\mathbf{C} \subseteq \mathbb{Q}^{d}$, let $\mathbf{V}$ be a vector space, and let us prove that $\overline{\mathbf{X}}$ is finitely generated where $\mathbf{X}=\mathbf{C} \cap \mathbf{V}$. Since $\mathbf{V}$ is generated by a finite set, we deduce that $\mathbf{V}$ and in particular $\mathbf{X}$ are definable in $\mathrm{FO}(\mathbb{Q},+, \leq, 0,1)$. Since this logic admits quantification elimination we deduce that there exists a quantifier free formula in this logic that denotes $\mathbf{X}$. Hence there exists a finite sequence $\left(A_{j}\right)_{1 \leq j \leq k}$ of finite sets $A_{j} \subseteq \mathbb{Q}^{d} \times\{>, \geq\} \times \mathbb{Q}$ such that $\mathbf{X}=\bigcup_{j=1}^{k} \mathbf{X}_{j}$ where:

$$
\mathbf{X}_{j}=\bigcap_{(\mathbf{h}, \#, b) \in A_{j}}\left\{\mathbf{x} \in \mathbb{Q}^{d} \mid \sum_{i=1}^{d} \mathbf{h}(i) \mathbf{x}(i) \# b\right\}
$$


We can assume without loss of generality that $\mathbf{X}_{j}$ is non empty. Let us introduce the following set $\mathbf{R}_{j}$ :

$$
\mathbf{R}_{j}=\bigcap_{(\mathbf{h}, \#, b) \in A_{j}}\left\{\mathbf{x} \in \mathbb{Q}^{d} \mid \sum_{i=1}^{d} \mathbf{h}(i) \mathbf{x}(i) \geq 0\right\}
$$

Lemma 3.4 shows that $\mathbf{R}_{j}$ is finitely generated. In particular $\mathbf{R}=$ $\sum_{j=1}^{k} \mathbf{R}_{j}$ is finitely generated. Thanks to Lemma 3.4, we deduce that $\mathbf{R}$ is closed. We are going to prove that $\overline{\mathbf{X}}=\mathbf{R}$. Let us first consider $\mathbf{x} \in \mathbf{X}$. For every $n \in \mathbb{N}$ we have $n \mathbf{x} \in \mathbf{X}$ since $\mathbf{X}$ is a conic set. Hence there exists $j \in\{1, \ldots, k\}$ such that $n \mathbf{x} \in \mathbf{X}_{j}$ for an infinite number of $n \in \mathbb{N}$. We deduce that $\mathbf{x} \in \mathbf{R}_{j}$. Thus $\mathbf{X} \subseteq \mathbf{R}$ and we have proved that $\overline{\mathbf{X}} \subseteq \mathbf{R}$ by minimality of the closure of $\mathbf{X}$. For the converse inclusion, let $\mathbf{r} \in \mathbf{R}$. There exists a sequence $\left(\mathbf{r}_{j}\right)_{1 \leq j \leq k}$ of vectors $\mathbf{r}_{j} \in \mathbf{R}_{j}$ such that $\mathbf{r}=\sum_{j=1}^{k} \mathbf{r}_{j}$. Since $\mathbf{X}_{j}$ is non empty, there exists $\mathbf{x}_{j} \in \mathbf{X}_{j}$. As $\mathbf{r}_{j} \in \mathbf{R}_{j}$ we deduce that $\mathbf{x}_{j}+\mathbb{Q}_{\geq 0} \mathbf{r}_{j} \subseteq \mathbf{X}_{j}$. Hence $\mathbf{x}+\sum_{j=1}^{k} \mathbb{Q}_{\geq 0} \mathbf{r}_{j} \subseteq \mathbf{X}$ where $\mathbf{x}=\sum_{j=1}^{k} \mathbf{x}_{j}$. In particular $\mathbf{x}+\mathbb{Q}_{\geq 0} \mathbf{r} \subseteq \mathbf{X}$. We deduce that for every $\epsilon \in \mathbb{Q}>0$ we have $\epsilon \mathbf{X}+\mathbf{r} \in \mathbf{X}$. Therefore $\mathbf{r} \in \overline{\mathbf{X}}$. We have proved the other inclusion. Thus $\bar{X}=\mathbf{R}$ is finitely generated.

Conversely, we prove by induction over $r$ that the conic sets $\mathbf{C} \subseteq \mathbb{Q}^{d}$ such that $\operatorname{rank}(\mathbf{C}-\mathbf{C}) \leq r$ and such that the conic set $\overline{\mathbf{C} \cap \mathbf{V}}$ is finitely generated for every vector space $\mathbf{V} \subseteq \mathbb{Q}^{d}$ are polytope. The case $r=0$ is immediate since in this case $\overline{\mathbf{C}}=\{\mathbf{0}\}$. Let us assume the induction proved for an integer $r \in \mathbb{N}$ and let us consider a conic set $\mathbf{C} \subseteq \mathbb{Q}^{d}$ such that $\operatorname{rank}(\mathbf{C}-\mathbf{C}) \leq r+1$ and such that the conic set $\overline{\mathbf{C} \cap \mathbf{V}}$ is finitely generated for every vector space $\mathbf{V} \subseteq \mathbb{Q}^{d}$. We introduce the vector space $\mathbf{W}=\mathbf{C}-\mathbf{C}$. Since $\overline{\mathbf{C}}=\overline{\mathbf{C} \cap \mathbf{V}}$ with $\mathbf{V}=\mathbb{Q}^{d}$, we deduce that $\overline{\mathbf{C}}$ is finitely generated. Lemma 3.4 shows that there exists a finite sequence $\left(\mathbf{h}_{j}\right)_{1 \leq j \leq k}$ of vectors $\mathbf{h}_{j} \in \mathbf{W} \backslash\{\mathbf{0}\}$ such that the following equality holds:

$$
\overline{\mathbf{C}}=\bigcap_{j=1}^{k}\left\{\mathbf{x} \in \mathbf{W} \mid \sum_{i=1}^{d} \mathbf{h}_{j}(i) \mathbf{x}(i) \geq 0\right\}
$$

Since $\operatorname{int}(\mathbf{C})=\operatorname{int}(\overline{\mathbf{C}})$ we get the following equality:

$$
\operatorname{int}(\mathbf{C})=\bigcap_{j=1}^{k}\left\{\mathbf{x} \in \mathbf{W} \mid \sum_{i=1}^{d} \mathbf{h}_{j}(i) \mathbf{x}(i)>0\right\}
$$

In particular int $(\mathbf{C})$ is definable in $\mathrm{FO}(\mathbb{Q},+, \leq, 0,1)$. As $\operatorname{int}(\mathbf{C}) \subseteq$ $\mathbf{C} \subseteq \overline{\mathbf{C}}$ we deduce the following decomposition where $\mathbf{W}_{j}=$ $\left\{\mathbf{w} \in \mathbf{W} \mid \sum_{i=1}^{d} \mathbf{h}_{j}(i) \mathbf{w}(i)=0\right\}$ :

$$
\mathbf{C}=\operatorname{int}(\mathbf{C}) \bigcup_{j=1}^{k}\left(\mathbf{C} \cap \mathbf{W}_{j}\right)
$$

Observe that $\mathbf{h}_{j} \in \mathbf{W} \backslash \mathbf{W}_{j}$ and in particular $\mathbf{W}_{j}$ is strictly included in $\mathbf{W}$. Thus $\operatorname{rank}\left(\mathbf{W}_{j}\right)<\operatorname{rank}(\mathbf{W}) \leq r+1$. Note that $\mathbf{C}_{j}=\mathbf{C} \cap \mathbf{W}_{j}$ is a conic set such that $\operatorname{rank}\left(\mathbf{C}_{j}-\mathbf{C}_{j}\right) \leq$

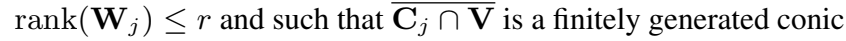
set for every vector space $\mathbf{V}$. Thus by induction $\mathbf{C}_{j}$ is definable in FO $(\mathbb{Q},+, \leq, 0,1)$. We deduce that $\mathbf{C}$ is polytope. We have proved the induction.

\section{Presburger Sets, Lambert Sets, And Petri Sets}

In this section we introduce the Presburger sets, Lambert Sets, and Petri Sets.

A periodic set is a subset $\mathbf{P} \subseteq \mathbb{Z}^{d}$ such that $\mathbf{0} \in \mathbf{P}$ and such that $\mathbf{P}+\mathbf{P} \subseteq \mathbf{P}$. A periodic set $\overline{\mathbf{P}}$ is said to be finitely generated if there exists a finite sequence $\mathbf{p}_{1}, \ldots, \mathbf{p}_{k}$ of vectors $\mathbf{p}_{j} \in \mathbf{P}$ such that $\mathbf{P}=\mathbb{N} \mathbf{p}_{1}+\cdots+\mathbb{N p}_{k}$. A subset $\mathbf{S} \subseteq \mathbb{Z}^{d}$ is called a Presburger

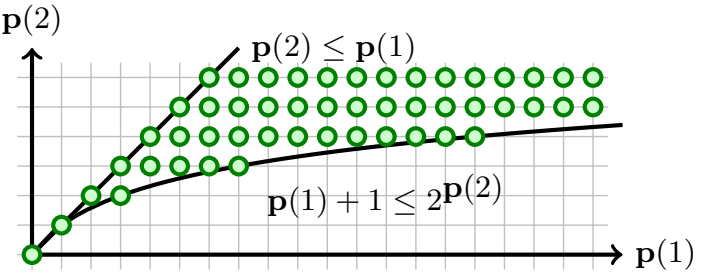

Figure 1. A polytope periodic set.

set if it can be denoted by a formula in the Presburger arithmetic $\mathrm{FO}(\mathbb{Z},+, \leq, 0,1)$. Let us recall [GS66] that a subset $\mathbf{S} \subseteq \mathbb{Z}^{d}$ is Presburger if and only if it is a finite union of sets $\mathbf{b}+\mathbf{P}$ where $\mathbf{b} \in \mathbb{Z}^{d}$ and $\mathbf{P} \subseteq \mathbb{Z}^{d}$ is a finitely generated periodic set. The class of Lambert sets is obtained by weakening the finiteness property of the periodic sets $\mathbf{P}$.

Definition 4.1. A periodic set $\mathbf{P}$ is said to be polytope if the conic set $\mathbb{Q} \geq 0 \mathbf{P}$ is polytope.

Remark 4.2. Every finitely generated periodic set $\mathbf{P}$ is polytope since in this case $\mathbb{Q} \geq 0 \mathbf{P}$ is a finitely generated conic set and in particular a polytope conic set.

Example 4.3. The periodic set $\mathbf{P}=\left\{\mathbf{p} \in \mathbb{N}^{2} \mid \mathbf{p}(1) \leq \sqrt{2} \mathbf{p}(2)\right\}$ is not polytope.

Example 4.4. The periodic set $\mathbf{P}=\left\{\mathbf{p} \in \mathbb{N}^{2} \mid \mathbf{p}(2) \leq\right.$ $\left.\mathbf{p}(1) \leq 2^{\mathbf{p}(2)}-1\right\}$ is represented in Figure 1. Observe that $\mathbb{Q}_{\geq 0} \mathbf{P}=\{\mathbf{0}\} \cup\left\{\mathbf{c} \in \mathbb{Q}_{>0}^{2} \mid \mathbf{p}(2) \leq \mathbf{p}(1)\right\}$ is a polytope conic set. Thus $\mathbf{P}$ is a polytope periodic set.

The following lemma shows that the class of polytope periodic sets is stable by finite intersections.

Lemma 4.5. We have $\left(\mathbb{Q}_{\geq 0} \mathbf{P}_{1}\right) \cap\left(\mathbb{Q}_{\geq 0} \mathbf{P}_{2}\right)=\mathbb{Q}_{\geq 0}\left(\mathbf{P}_{1} \cap \mathbf{P}_{2}\right)$ for every periodic sets $\mathbf{P}_{1}, \mathbf{P}_{2} \subseteq \mathbb{Z}^{d}$.

Proof. Observe that $\mathbf{P}_{1} \subseteq \mathbb{Q}_{\geq 0} \mathbf{P}_{1}$ and $\mathbf{P}_{2} \subseteq \mathbb{Q}_{\geq 0} \mathbf{P}_{2}$. Hence $\mathbf{P}_{1} \cap \mathbf{P}_{2} \subseteq \mathbf{C}$ where $\mathbf{C}=\left(\mathbb{Q} \geq 0 \mathbf{P}_{1}\right) \cap\left(\mathbb{Q}_{\geq 0} \mathbf{P}_{2}\right)$. As $\mathbf{C}$ is a conic set we deduce that $\mathbb{Q} \geq 0,\left(\mathbf{P}_{1} \cap \mathbf{P}_{2}\right) \subseteq \mathbf{C}$. For the converse inclusion. Let $\mathbf{c} \in \mathbf{C}$. Since $\mathbf{c} \in \mathbb{Q}_{\geq 0} \mathbf{P}_{1}$, there exists $\lambda_{1} \in \mathbb{Q}_{\geq 0}$ such that $\mathbf{c} \in \lambda_{1} \mathbf{P}_{1}$. Symmetrically there exists $\lambda_{2} \in \mathbb{Q} \geq 0$ such that $\mathbf{c} \in \lambda_{2} \mathbf{P}_{2}$. Let $n_{1}, n_{2} \in \mathbb{N}_{>0}$ such that $n_{1} \lambda_{1} \in \mathbb{N}$ and $n_{2} \lambda_{2} \in \mathbb{N}$. Let $n=n_{1} n_{2}$ and observe that $n \mathbf{c} \in n_{2}\left(n_{1} \lambda_{1}\right) \mathbf{P}_{1} \subseteq \mathbf{P}_{1}$ since $\mathbf{P}_{1}$ is a periodic set. Symmetrically $n \mathbf{c} \in \mathbf{P}_{2}$. We have proved that $n \mathbf{c} \in \mathbf{P}_{1} \cap \mathbf{P}_{2}$. Thus $\mathbf{c} \in \mathbb{Q}_{\geq 0}\left(\mathbf{P}_{1} \cap \mathbf{P}_{2}\right)$ and we get the other inclusion.

Lambert sets and Petri sets are central in this paper.

Definition 4.6. A Lambert set $\mathbf{L} \subseteq \mathbb{Z}^{d}$ is a finite union of sets $\mathbf{b}+\mathbf{P}$ where $\mathbf{b} \in \mathbb{Z}^{d}$ and $\mathbf{P} \subseteq \mathbb{Z}^{d}$ is a polytope periodic set.

Definition 4.7. A Petri set is a subset $\mathbf{X} \subseteq \mathbb{Z}^{d}$ such that $\mathbf{X} \cap \mathbf{S}$ is a Lambert set for every Presburger set $\mathbf{S} \subseteq \mathbb{Z}^{d}$.

Example 4.8. Let us consider the periodic set $\mathbf{P}=\{(0,0)\} \cup$ $\left\{\left(2^{n}, 1\right) \mid n \in \mathbb{N}\right\} \cup\left((1,2)+\mathbb{N}^{2}\right)$. Observe that $\mathbb{Q} \geq 0$ P is the polytope conic set $\{(0,0)\} \cup \mathbb{Q}_{>0}^{2}$. We deduce that $\mathbf{P}$ is a Lambert set. Note that $\mathbf{P}$ is not a Petri set since $\mathbf{P} \cap(\mathbb{N} \times\{1\})=\left\{\left(2^{n}, 1\right) \mid\right.$ $n \in \mathbb{N}\}$ is not a Lambert set.

The class of Petri sets is between the class of Presburger sets and the class of Lambert sets. The previous Example 4.8 shows that the class of Petri sets is strictly included in the class of Lambert sets. The strict inclusion of the class of Presburger sets into the class of 
Petri sets will be a direct consequence of a stronger result proved in this paper. In fact the reachability relation of a Vector Addition System is proved to be a Petri relation and we know that in general such a relation is not Presburger.

\section{Linearizations}

The linearization of a periodic set $\mathbf{P} \subseteq \mathbb{Z}^{d}$ is the periodic set $\operatorname{lin}(\mathbf{P})$ defined by the following equality:

$$
\operatorname{lin}(\mathbf{P})=(\mathbf{P}-\mathbf{P}) \cap \overline{\mathbb{Q} \geq 0} \overline{\mathbf{P}}
$$

Lemma 5.1. The linearization of a polytope periodic set is finitely generated.

Proof. Let $\mathbf{V}$ be the vector space generated by $\mathbf{P}$ and let us introduce the conic set $\mathbf{C}=\overline{\mathbb{Q}} \geq 0 \mathbf{P}$. Note that $\mathbb{Q} \geq 0 \mathbf{P} \subseteq \mathbf{V}$ and since $\mathbf{V}$ is closed we get $\mathbf{C} \subseteq \mathbf{V}$. As $\mathbb{Q}_{\geq 0} \mathbf{P}$ is a polytope conic set we deduce that $\mathbf{C}$ is finitely generated. Hence there exists $\mathbf{c}_{1}, \ldots, \mathbf{c}_{k} \in \mathbf{C}$ such that $\mathbf{C}=\mathbb{Q}_{\geq 0} \mathbf{c}_{1}+\cdots+\mathbb{Q}_{\geq 0} \mathbf{c}_{k}$. As $\mathbf{c}_{j} \in \mathbf{C} \subseteq \mathbf{V}=\mathbb{Q}_{\geq 0} \mathbf{P}-\mathbb{Q}_{\geq 0} \mathbf{P}$, by replacing $\mathbf{c}_{j}$ by a vector in $\mathbb{N}_{>0} \mathbf{c}_{j}$ we can assume that $\mathbf{c}_{j} \in \mathbf{P}-\mathbf{P}$ for every $j \in\{1, \ldots, k\}$.

We introduce the following set $\mathbf{R}$ :

$$
\mathbf{R}=\left\{\mathbf{r} \in \mathbf{P}-\mathbf{P} \mid \mathbf{r}=\sum_{j=1}^{k} \lambda_{j} \mathbf{c}_{j} \quad \lambda_{j} \in \mathbb{Q} 0 \leq \lambda_{j}<1\right\}
$$

We observe that every vector $\mathbf{r} \in \mathbf{R}$ satisfies $\|\mathbf{r}\|_{\infty} \leq s$ where $s=\sum_{j=1}^{k}\left\|\mathbf{c}_{j}\right\|_{\infty}$. Hence $\mathbf{R} \subseteq\{-s, \ldots, s\}^{d}$ and we deduce that $\mathbf{R}$ is finite.

Let $\mathbf{L}$ be the periodic set generated by the finite set $\mathbf{R} \cup$ $\left\{\mathbf{c}_{1}, \ldots, \mathbf{c}_{k}\right\}$. Since this finite set is included in $\operatorname{lin}(\mathbf{P})$ we deduce that $\mathbf{L} \subseteq \operatorname{lin}(\mathbf{P})$. Let us prove the converse inclusion. Let $\mathbf{x} \in \operatorname{lin}(\mathbf{P})$. Since $\mathbf{x} \in \mathbf{C}$, there exists a sequence $\left(\mu_{j}\right)_{1 \leq j \leq k}$ of rational elements $\mu_{j} \in \mathbb{Q}_{\geq 0}$ such that $\mathbf{x}=\sum_{j=1}^{k} \mu_{j} \mathbf{c}_{j}$. Let us introduce $n_{j} \in \mathbb{N}$ such that $\lambda_{j}=\mu_{j}-n_{j}$ satisfies $0 \leq \lambda_{j}<1$. Let $\mathbf{r}=\sum_{j=1}^{k} \lambda_{j} \mathbf{c}_{j}$. As $\mathbf{r}=\mathbf{x}-\sum_{j=1}^{k} n_{j} \mathbf{c}_{j}$ we get $\mathbf{r} \in \mathbf{P}-\mathbf{P}$. Thus $\mathbf{r} \in \mathbf{R}$. From $\mathbf{x}=\mathbf{r}+\sum_{j=1}^{k} n_{j} \mathbf{c}_{j}$ we get $\mathbf{x} \in \mathbf{L}$. We have proved that $\operatorname{lin}(\mathbf{P})=\mathbf{L}$ is finitely generated.

We observe that if the intersection $\left(\mathbf{b}_{1}+\mathbf{P}_{1}\right) \cap\left(\mathbf{b}_{2}+\mathbf{P}_{2}\right)$ is empty where $\mathbf{b}_{1}, \mathbf{b}_{2} \in \mathbb{Z}^{d}$ and $\mathbf{P}_{1}, \mathbf{P}_{2} \subseteq \mathbb{Z}^{d}$ are two polytope periodic sets then the intersection $\left(\mathbf{b}_{1}+\operatorname{lin}\left(\mathbf{P}_{1}\right)\right) \cap\left(\mathbf{b}_{2}+\operatorname{lin}\left(\mathbf{P}_{2}\right)\right)$ may be non empty. In this section we show that a dimension is strictly decreasing.

Let us first introduce our definition of dimension. The dimension $\operatorname{dim}(\mathbf{X})$ of a non-empty set $\mathbf{X} \subseteq \mathbb{Z}^{d}$ is the minimal integer $r \in\{0, \ldots, d\}$ such that there exists $k \in \mathbb{N}_{>0}$, a sequence $\left(\mathbf{b}_{j}\right)_{1 \leq j \leq k}$ of vectors $\mathbf{b}_{j} \in \mathbb{Z}^{d}$, and a sequence $\left(\mathbf{V}_{j}\right)_{1 \leq j \leq k}$ of vector spaces $\mathbf{V}_{j} \subseteq \mathbb{Q}^{d}$ such that $\operatorname{rank}\left(\mathbf{V}_{j}\right) \leq r$ and such that $\mathbf{X} \subseteq \bigcup_{j=1}^{k} \mathbf{b}_{j}+\mathbf{V}_{j}$. The dimension of the empty set is defined by $\operatorname{dim}(\emptyset)=-1$.

In the reminder of this section we prove the following Theorem 5.2. All other results or definitions introduced in this section are not used in the sequel.

Theorem 5.2. Let $\mathbf{b}_{1}, \mathbf{b}_{2} \in \mathbb{Z}^{d}$ and let $\mathbf{P}_{1}, \mathbf{P}_{2}$ be two polytope periodic sets such that the intersection $\left(\mathbf{b}_{1}+\mathbf{P}_{1}\right) \cap\left(\mathbf{b}_{2}+\mathbf{P}_{2}\right)$ is empty. The intersection $\mathbf{X}=\left(\mathbf{b}_{1}+\operatorname{lin}\left(\mathbf{P}_{1}\right)\right) \cap\left(\mathbf{b}_{2}+\operatorname{lin}\left(\mathbf{P}_{2}\right)\right)$ satisfies:

$$
\operatorname{dim}(\mathbf{X})<\max \left\{\operatorname{dim}\left(\mathbf{b}_{1}+\mathbf{P}_{1}\right), \operatorname{dim}\left(\mathbf{b}_{2}+\mathbf{P}_{2}\right)\right\}
$$

We first characterize the dimension of a periodic set.

Lemma 5.3. Let $\mathbf{V}$ be the vector space generated by a periodic set P. Then $\operatorname{rank}(\mathbf{V})=\operatorname{dim}(\mathbf{P})$.
Proof. Let $\mathbf{P}$ be a periodic set and let us first prove by induction over $k \in \mathbb{N}_{>0}$ that $\mathbf{P} \subseteq \bigcup_{j=1}^{k} \mathbf{V}_{j}$ implies that there exists $j \in\{1, \ldots, k\}$ such that $\mathbf{P} \subseteq \mathbf{V}_{j}$ for every sequence $\left(\mathbf{V}_{j}\right)_{1 \leq j \leq k}$ of vector spaces $\mathbf{V}_{j} \subseteq \mathbb{Q}^{d}$. The case $k=1$ is immediate. Assume the property proved for an integer $k \in \mathbb{N}_{>0}$ and let us assume that $\mathbf{P} \subseteq \bigcup_{j=1}^{k+1} \mathbf{V}_{j}$. If $\mathbf{P} \subseteq \mathbf{V}_{k+1}$ the property is proved. So we can assume that there exists $\mathbf{p} \in \mathbf{P} \backslash \mathbf{V}_{k+1}$. Let us prove that $\mathbf{P} \subseteq \bigcup_{j=1}^{k} \mathbf{V}_{j}$. We consider $\mathbf{x} \in \mathbf{P}$. Observe that if $\mathbf{x} \notin \mathbf{V}_{k+1}$ then $\mathbf{x} \in \bigcup_{j=1}^{k} \mathbf{V}_{j}$. So we can assume that $\mathbf{x} \in \mathbf{V}_{k+1}$. We observe that $\mathbf{p}+n \mathbf{x} \in \mathbf{P}$ for every $n \in \mathbb{N}$ since the set $\mathbf{P}$ is periodic. We deduce that there exists $j \in\{1, \ldots, k+1\}$ such that $\mathbf{p}+n \mathbf{x} \in \mathbf{V}_{j}$. Naturally this integer $j$ depends on $n$. However, since $\{1, \ldots, k+1\}$ is finite whereas $\mathbb{N}$ is infinite, there exists $j \in\{1, \ldots, k+1\}$ and $n<n^{\prime}$ in $\mathbb{N}$ such that $\mathbf{p}+n \mathbf{x}$ and $\mathbf{p}+n^{\prime} \mathbf{x}$ are both in $\mathbf{V}_{j}$. As $\mathbf{V}_{j}$ is a vector space, we deduce that $n^{\prime}(\mathbf{p}+n \mathbf{x})-n\left(\mathbf{p}+n^{\prime} \mathbf{x}\right)$ is in $\mathbf{V}_{j}$. Hence $\mathbf{p} \in \mathbf{V}_{j}$. As $\mathbf{p} \notin \mathbf{V}_{k+1}$ we deduce that $j \neq k+1$. As $\mathbf{V}_{j}$ is a vector space we deduce that $\left(\mathbf{p}+n^{\prime} \mathbf{x}\right)-(\mathbf{p}+n \mathbf{x}) \in \mathbf{V}_{j}$. Hence $\mathbf{x} \in \mathbf{V}_{j}$. We have proved that $\mathbf{x} \in \bigcup_{j=1}^{k} \mathbf{V}_{j}$. Thus $\mathbf{P} \subseteq \bigcup_{j=1}^{k} \mathbf{V}_{j}$ and by induction there exists $j \in\{1, \ldots, k\}$ such that $\mathbf{P} \subseteq \mathbf{V}_{j}$. We have proved the induction.

Now, let us prove the lemma. We consider a periodic set $\mathbf{P}$ and we let $\mathbf{V}$ be the vector space generated by this set. Since $\mathbf{P} \subseteq \mathbf{V}$ we deduce that $\operatorname{dim}(\mathbf{P}) \leq \operatorname{rank}(\mathbf{V})$. For the converse inclusion, since $\mathbf{P}$ is non empty we deduce that $\mathbf{P} \subseteq \bigcup_{j=1}^{k} \mathbf{b}_{j}+\mathbf{V}_{j}$ where $k \in \mathbb{N}_{>0}, \mathbf{b}_{j} \in \mathbb{Z}^{d}$ and $\mathbf{V}_{j} \subseteq \mathbb{Q}^{d}$ is a vector space such that $\operatorname{rank}\left(\mathbf{V}_{j}\right) \leq \operatorname{dim}(\mathbf{P})$. Let us consider the set $J=\{j \in$ $\left.\{1, \ldots, k\} \mid \mathbf{b}_{j} \in \mathbf{V}_{j}\right\}$ and let us prove that $\mathbf{P} \subseteq \bigcup_{j \in J} \mathbf{V}_{j}$. Let $\mathbf{p} \in \mathbf{P}$ and $n \in \mathbb{N}$. Since $n \mathbf{p} \in \mathbf{P}$ there exists $j \in\{1, \ldots, k\}$ such that $n \mathbf{p} \in \mathbf{b}_{j}+\mathbf{V}_{j}$. Hence there exists $j \in\{1, \ldots, k\}$ and $n<n^{\prime}$ in $\mathbb{N}$ such that $n \mathbf{p}$ and $n^{\prime} \mathbf{p}$ are both in $\mathbf{b}_{j}+\mathbf{V}_{j}$. As $\mathbf{V}_{j}$ is a vector space we deduce that $n^{\prime} \mathbf{p}-n \mathbf{p} \in \mathbf{V}_{j}$. Thus $\mathbf{p} \in \mathbf{V}_{j}$. Moreover as $\mathbf{b}_{j} \in n \mathbf{p}-\mathbf{V}_{j} \subseteq \mathbf{V}_{j}$ we deduce that $j \in J$. We have prove the inclusion $\mathbf{P} \subseteq \bigcup_{j \in J} \mathbf{V}_{j}$. From the previous paragraph we deduce that there exists $j \in J$ such that $\mathbf{P} \subseteq \mathbf{V}_{j}$. By minimality of the vector space generated by $\mathbf{P}$ we get $\overline{\mathbf{V}} \subseteq \mathbf{V}_{j}$. Hence $\operatorname{rank}(\mathbf{V}) \leq \operatorname{rank}\left(\mathbf{V}_{j}\right)$. Since $\operatorname{rank}\left(\mathbf{V}_{j}\right) \leq \operatorname{dim}(\overline{\mathbf{P}})$ we have proved the inequality $\operatorname{rank}(\mathbf{V}) \leq \operatorname{dim}(\mathbf{P})$.

Next we prove a separation property.

Lemma 5.4. Let $\mathbf{C}_{\leq}$and $\mathbf{C}_{\geq}$be two finitely generated conic sets that generates the same vector space $\mathbf{V}$ and such that the vector space generated by $\mathbf{C}_{\leq} \cap \mathbf{C}_{\geq}$is strictly included in $\mathbf{V}$. Then there exists a vector $\mathbf{h} \in \mathbf{V} \backslash\{\mathbf{0}\}$ such that for every $\# \in\{\leq, \geq\}$, we have:

$$
\mathbf{C}_{\#} \subseteq\left\{\mathbf{v} \in \mathbf{V} \mid \sum_{i=1}^{d} \mathbf{h}(i) \mathbf{v}(i) \# 0\right\}
$$

Proof. Lemma 3.4 shows that there exists two finite sets $\mathbf{H}_{\leq}, \mathbf{H}_{\geq}$ included in $\mathbf{V} \backslash\{\mathbf{0}\}$ such that:

$$
\begin{gathered}
\mathbf{C}_{\#}=\bigcap_{\mathbf{h} \in \mathbf{H}_{\#}}\left\{\mathbf{v} \in \mathbf{V} \mid \sum_{i=1}^{d} \mathbf{h}(i) \mathbf{v}(i) \geq 0\right\} \\
\operatorname{int}\left(\mathbf{C}_{\#}\right)=\bigcap_{\mathbf{h} \in \mathbf{H}_{\#}}\left\{\mathbf{v} \in \mathbf{V} \mid \sum_{i=1}^{d} \mathbf{h}(i) \mathbf{v}(i)>0\right\}
\end{gathered}
$$

Assume by contradiction that the intersection $\operatorname{int}\left(\mathbf{C}_{\leq}\right) \cap \operatorname{int}\left(\mathbf{C}_{\geq}\right)$ is non empty and let $\mathbf{c}$ be a vector in this set. Observe that there exists $\epsilon \in \mathbb{Q}_{>0}$ such that $\mathbf{c}+\mathbf{v} \in \mathbf{C}_{\leq} \cap \mathbf{C}_{\geq}$for every $\mathbf{v} \in \mathbf{V}$ such that $\|\mathbf{v}\|_{\infty}<\epsilon$. We deduce that the vector space generated by $\mathbf{C}_{\leq} \cap \mathbf{C}_{\geq}$contains $\mathbf{V}$ and we get a contradiction. 
We deduce that the following intersection is empty where $\mathbf{H}=$ $\mathbf{H}_{\leq} \cup \mathbf{H}_{\geq}$

$$
\bigcap_{\mathbf{h} \in \mathbf{H}}\left\{\mathbf{v} \in \mathbf{V} \mid \sum_{i=1}^{d} \mathbf{h}(i) \mathbf{v}(i)>0\right\}
$$

The Farkas's lemma [Sch87] shows that there exists a nonzero function $f: \mathbf{H} \rightarrow \mathbb{Q}_{\geq 0}$ such that $\sum_{\mathbf{h} \in \mathbf{H}} f(\mathbf{h}) \mathbf{h}=\mathbf{0}$. Let us introduce $\mathbf{a}=\sum_{\mathbf{h} \in \mathbf{H}_{>}} f(\mathbf{h}) \mathbf{h}$ and $\mathbf{b}=\sum_{\mathbf{h} \in \mathbf{H} \backslash \mathbf{H}_{>}} f(\mathbf{h}) \mathbf{h}$. Assume by contradiction that $\mathbf{a}=\mathbf{0}$. Since $\mathbf{a}+\mathbf{b}=\mathbf{0}$ we deduce that $\mathbf{b}=\mathbf{0}$. As $f$ is not the zero function, there exists $\mathbf{h} \in \mathbf{H}$ such that $f(\mathbf{h}) \neq 0$. Note that either $\mathbf{h} \in \mathbf{H}_{\geq}$or $\mathbf{h} \in \mathbf{H} \backslash \mathbf{H}_{\geq}$. In the first case we deduce that $\operatorname{int}\left(\mathbf{C}_{\geq}\right)$is empty and in the second case we deduce that $\operatorname{int}\left(\mathbf{C}_{\leq}\right)$is empty. Since both cases are impossible we get a contradiction. Thus $\mathbf{a} \neq \mathbf{0}$. For every $\mathbf{c} \in \operatorname{int}\left(\mathbf{C}_{\geq}\right)$we have $\sum_{i=1}^{d} \mathbf{a}(i) \mathbf{c}(i) \geq 0$. Since the set $\left\{\mathbf{c} \in \mathbb{Q}^{d} \mid \sum_{i=1}^{d} \mathbf{a}(i) \mathbf{c}(i) \geq 0\right\}$ is closed we deduce that for every $\mathbf{c} \in \overline{\operatorname{int}\left(\mathbf{C}_{\geq}\right)}=\mathbf{C}_{\geq}$the same inequality holds. Now let us consider $\mathbf{c} \in \operatorname{int}\left(\mathbf{C}_{\leq}\right)$. In this case $\sum_{i=1}^{d} \mathbf{b}(i) \mathbf{c}(i) \geq 0$. Since $\mathbf{a}+\mathbf{b}=\mathbf{0}$ we get $\sum_{i=1}^{d} \mathbf{a}(i) \mathbf{c}(i) \leq 0$. We deduce that this inequality holds for every $\mathbf{c} \in \mathbf{C}_{\leq}$.

Remark 5.5. The previous Lemma 5.4 is wrong if we remove the finitely generated condition on the conic sets $\mathbf{C}_{\leq}$and $\mathbf{C}_{\geq}$. In fact let us consider the conic sets $\mathbf{C}_{\leq}=\left\{\mathbf{x} \in \mathbb{Q}_{\geq 0}^{2} \mid \mathbf{x}(1) \leq \sqrt{2} \mathbf{x}(2)\right\}$ and $\mathbf{C}_{\geq}=\left\{\mathbf{x} \in \mathbb{Q}_{\geq 0}^{2} \mid \mathbf{x}(2) \geq \sqrt{2} \mathbf{x}(2)\right\}$. Observe that $\mathbf{C}_{\leq} \cap \mathbf{C}_{\geq}=\{\mathbf{0}\}$. Hence the vector space generated by the intersection is strictly included in $\mathbb{Q}^{2}$. However there does not exist a vector $\mathbf{a} \in \mathbb{Q}^{2} \backslash\{\mathbf{0}\}$ satisfying the separation property required by Lemma 5.4. This problem can be overcome by introducing the vector spaces of $\mathbb{R}^{d}$. We do not introduce this extension to simplify the presentation.

We can now provide a proof of Theorem 5.2. We consider two vectors $\mathbf{b}_{1}, \mathbf{b}_{2} \in \mathbb{Z}^{d}$ and two periodic sets $\mathbf{P}_{1}, \mathbf{P}_{2} \subseteq \mathbb{Z}^{d}$ such that $\left(\mathbf{b}_{1}+\mathbf{P}_{1}\right) \cap\left(\mathbf{b}_{2}+\mathbf{P}_{2}\right)=\emptyset$. We introduce the intersection $\mathbf{X}=\left(\mathbf{b}_{1}+\operatorname{lin}\left(\mathbf{P}_{1}\right)\right) \cap\left(\mathbf{b}_{2}+\operatorname{lin}\left(\mathbf{P}_{2}\right)\right)$. Observe that if $\mathbf{X}$ is empty the theorem is proved. So we can assume that there exists $\mathbf{a}$ vector $\mathbf{b}$ in this intersection. Let us denote by $\mathbf{V}_{1}$ and $\mathbf{V}_{2}$ the vector spaces generated by $\mathbf{P}_{1}$ and $\mathbf{P}_{2}$. Lemma 5.3 shows that $\operatorname{rank}\left(\mathbf{V}_{j}\right)=\operatorname{dim}\left(\mathbf{P}_{j}\right)$ and from $\operatorname{dim}\left(\mathbf{b}_{j}+\mathbf{P}_{j}\right)=\operatorname{dim}\left(\mathbf{P}_{j}\right)$ we deduce that $\operatorname{dim}\left(\mathbf{b}_{j}+\mathbf{P}_{j}\right)=\operatorname{rank}\left(\mathbf{V}_{j}\right)$. As $\mathbf{X}$ is included in $\mathbf{b}+\mathbf{V}$ where $\mathbf{V}=\mathbf{V}_{1} \cap \mathbf{V}_{2}$, we deduce that if $\mathbf{V}$ is strictly included in $\mathbf{V}_{j}$ for one $j \in\{1,2\}$ then $\operatorname{dim}(\mathbf{X}) \leq \operatorname{rank}(\mathbf{V})<\operatorname{rank}\left(\mathbf{V}_{j}\right)=$ $\operatorname{dim}\left(\mathbf{b}_{j}+\mathbf{P}_{j}\right)$ and the theorem is proved. So we can assume that $\mathbf{V}_{1}=\mathbf{V}_{2}=\mathbf{V}$. Let us consider the conic sets $\mathbf{C}_{1}=\overline{\mathbb{Q}_{\geq 0} \mathbf{P}_{1}}$ and $\mathbf{C}_{2}=\overline{\mathbb{Q} \geq 0} \mathbf{P}_{2}$. Since $\mathbf{P}_{1}$ and $\mathbf{P}_{2}$ are polytope periodic sets, we deduce that $\mathbf{C}_{1}$ and $\mathbf{C}_{2}$ are finitely generated conic sets. Note that $\mathbf{C}_{1}, \mathbf{C}_{2} \subseteq \mathbf{V}$. We introduce the intersection $\mathbf{C}=\mathbf{C}_{1} \cap \mathbf{C}_{2}$.

Assume by contradiction that the vector space generated by $\mathbf{C}$ is equal to $\mathbf{V}$. Let us consider a vector $\mathbf{c}$ in the interior of $\mathbf{C}$. The characterization given by Lemma 3.4 shows that in this case $\operatorname{int}(\mathbf{C})=\operatorname{int}\left(\mathbf{C}_{1}\right) \cap \operatorname{int}\left(\mathbf{C}_{2}\right)$. Since $\operatorname{int}\left(\mathbf{C}_{j}\right)=\operatorname{int}\left(\mathbb{Q} \geq 0 . \mathbf{P}_{j}\right)$ we deduce that $\mathbf{c} \in\left(\mathbb{Q}_{\geq 0} \mathbf{P}_{1}\right) \cap\left(\mathbb{Q}_{\geq 0} \mathbf{P}_{2}\right)$. Lemma 4.5 shows that $\mathbf{c} \in \mathbb{Q}_{\geq 0}\left(\mathbf{P}_{1} \cap \mathbf{P}_{2}\right)$. By replacing $\mathbf{c}$ be a vector in $\mathbb{N}_{>0} \mathbf{c}$ we can assume that $\mathbf{c} \in \mathbf{P}_{1} \cap \mathbf{P}_{2}$.

Let us prove that there exists $k_{1} \in \mathbb{N}$ such that $\mathbf{b}+k_{1} \mathbf{c} \in$ $\mathbf{b}_{1}+\mathbf{P}_{1}$. From $\mathbf{b} \in \mathbf{b}_{1}+\operatorname{lin}\left(\mathbf{P}_{1}\right)$ we deduce that there exists $\mathbf{p}_{1}, \mathbf{p}_{1}^{\prime} \in \mathbf{P}_{1}$ such that $\mathbf{b}=\mathbf{b}_{1}+\mathbf{p}_{1}-\mathbf{p}_{1}^{\prime}$. Since $-\mathbf{p}_{1}^{\prime}$ is in the vector space generated by $\mathbf{C}$ and $\mathbf{c}$ is in the interior of $\mathbf{C}$, there exists $n_{1} \in \mathbb{N}$ large enough such that $n_{1} \mathbf{c}+\left(-\mathbf{p}_{1}^{\prime}\right) \in \mathbf{C}_{1}$. Hence there exists $n_{1}^{\prime} \in \mathbb{N}_{>0}$ such that $n_{1} n_{1}^{\prime} \mathbf{c}-n_{1}^{\prime} \mathbf{p}_{1}^{\prime} \in \mathbf{P}_{1}$. Thus $n_{1} n_{1}^{\prime} \mathbf{c}-\mathbf{p}_{1}^{\prime} \in\left(n_{1}^{\prime}-1\right) \mathbf{p}_{1}^{\prime}+\mathbf{P}_{1} \subseteq \mathbf{P}_{1}$. Hence $\mathbf{b}+k_{1} \mathbf{c} \in \mathbf{b}_{1}+\mathbf{P}_{1}$ with $k_{1}=n_{1} n_{1}^{\prime}$.

Symmetrically we deduce that there exists $k_{2} \in \mathbb{N}$ such that $\mathbf{b}+k_{2} \mathbf{c} \in \mathbf{b}_{2}+\mathbf{P}_{2}$. We have proved that $\mathbf{b}+\left(k_{1}+k_{2}\right) \mathbf{c} \in$
$\left(\mathbf{b}_{1}+\mathbf{P}_{1}\right) \cap\left(\mathbf{b}_{2}+\mathbf{P}_{2}\right)$ and we get a contradiction since this intersection is supposed to be empty.

We deduce that the vector space generated by $\mathbf{C}$ is strictly included in $\mathbf{V}$. Lemma 5.4 shows that there exists a vector $\mathbf{h} \in$ $\mathbf{V} \backslash\{\mathbf{0}\}$ such that:

$$
\begin{aligned}
& \mathbf{C}_{1} \subseteq\left\{\mathbf{v} \in \mathbf{V} \mid \sum_{i=1}^{d} \mathbf{h}(i) \mathbf{v}(i) \geq 0\right\} \\
& \mathbf{C}_{2} \subseteq\left\{\mathbf{v} \in \mathbf{V} \mid \sum_{i=1}^{d} \mathbf{h}(i) \mathbf{v}(i) \leq 0\right\}
\end{aligned}
$$

By replacing $\mathbf{h}$ by a vector in $\mathbb{N}_{>0} \mathbf{h}$ we can assume that $\mathbf{h} \in \mathbb{Z}^{d}$. Now let us consider $\mathbf{x} \in \mathbf{X}$. Since $\mathbf{x}-\mathbf{b}_{1} \in \mathbf{C}_{1}$ we deduce that $\sum_{i=1}^{d} \mathbf{h}(i)\left(\mathbf{x}(i)-\mathbf{b}_{1}(i)\right) \geq 0$ and since $\mathbf{x}-\mathbf{b}_{2} \in \mathbf{C}_{2}$ we deduce that $\sum_{i=1}^{d} \mathbf{h}(i)\left(\mathbf{x}(i)-\mathbf{b}_{2}(i)\right) \leq 0$. We introduce the integers $z_{1}=\sum_{i=1}^{d} \mathbf{h}(i) \mathbf{b}_{1}(i)$ and $z_{2}=\sum_{i=1}^{d} \mathbf{h}(i) \mathbf{b}_{2}(i)$. We have proved that $\mathbf{X}$ can be decomposed into a finite union of slices $\mathbf{X}=\bigcup_{z=z_{1}}^{z_{2}} \mathbf{X}_{z}$ where:

$$
\mathbf{X}_{z}=\left\{\mathbf{x} \in \mathbf{X} \mid \sum_{i=1}^{d} \mathbf{h}(i) \mathbf{x}(i)=z\right\}
$$

Let us prove that $\operatorname{dim}\left(\mathbf{X}_{z}\right)<\operatorname{rank}(\mathbf{V})$. If $\mathbf{X}_{z}$ is empty the relation is immediate. If $\mathbf{X}_{z}$ is non empty let us consider $\mathbf{x} \in \mathbf{X}_{z}$ and observe that $\mathbf{X}_{z} \subseteq \mathbf{x}+\mathbf{W}$ where:

$$
\mathbf{W}=\left\{\mathbf{v} \in \mathbf{V} \mid \sum_{i=1}^{d} \mathbf{a}(i) \mathbf{v}(i)=0\right\}
$$

Note that $\mathbf{h} \in \mathbf{V} \backslash \mathbf{W}$. We deduce that $\mathbf{W}$ is strictly included in $\mathbf{V}$ and in particular $\operatorname{rank}(\mathbf{W})<\operatorname{rank}(\mathbf{V})$. Hence $\operatorname{dim}\left(\mathbf{X}_{z}\right)<$ $\operatorname{rank}(\mathbf{V})$.

From $\mathbf{X}=\bigcup_{z=z_{1}}^{z_{2}} \mathbf{X}_{z}$ and $\operatorname{dim}\left(\mathbf{X}_{z}\right)<\operatorname{rank}(\mathbf{V})$ for every $z$, we deduce that $\operatorname{dim}(\mathbf{X})<\operatorname{rank}(\mathbf{V})$ and the theorem is proved.

\section{Presburger Invariants}

Given a relation $R$ over $\mathbb{Z}^{d}$ and two sets $\mathbf{X}, \mathbf{Y} \subseteq \mathbb{Z}^{d}$ we introduce the forward image post ${ }_{R}(\mathbf{X})$ and the backward image $\operatorname{pre}_{R}(\mathbf{Y})$ defined by the following equalities:

$$
\begin{cases}\operatorname{post}_{R}(\mathbf{X}) & =\bigcup_{\mathbf{x} \in \mathbf{X}}\left\{\mathbf{y} \in \mathbb{Z}^{d} \mid(\mathbf{x}, \mathbf{y}) \in R\right\} \\ \operatorname{pre}_{R}(\mathbf{Y}) & =\bigcup_{\mathbf{y} \in \mathbf{Y}}\left\{\mathbf{x} \in \mathbb{Z}^{d} \mid(\mathbf{x}, \mathbf{y}) \in R\right\}\end{cases}
$$

We say that a set $\mathbf{X} \subseteq \mathbb{Z}^{d}$ is a forward invariant for $R$ if post $_{R}(\mathbf{X}) \subseteq \mathbf{X}$ and we say that a set $\mathbf{Y} \subseteq \mathbb{Z}^{d}$ is a backward invariant for $R$ if $\operatorname{pre}_{R}(\mathbf{Y}) \subseteq \mathbf{Y}$. In the reminder of this section we prove the following Theorem 6.1. All other results or definitions introduced in this section are not used in the sequel.

Theorem 6.1. Let $R^{*}$ be a reflexive and transitive Petri relation over $\mathbb{Z}^{d}$ and let $\mathbf{X}, \mathbf{Y} \subseteq \mathbb{Z}^{d}$ be two Presburger sets such that $R^{*} \cap(\mathbf{X} \times \mathbf{Y})$ is empty. There exists a partition of $\mathbb{Z}^{d}$ into a Presburger forward invariant that contains $\mathbf{X}$ and a Presburger backward invariant that contains $\mathbf{Y}$.

We first prove the following lemma.

Lemma 6.2. The sets $\operatorname{post}_{R}(\mathbf{X})$ and $\operatorname{pre}_{R}(\mathbf{Y})$ are Petri sets for every Petri relation $R \subseteq \mathbb{Z}^{d} \times \mathbb{Z}^{d}$ and for every Presburger sets $\mathbf{X}, \mathbf{Y} \subseteq \mathbb{Z}^{d}$

Proof. Let us first prove that $\operatorname{post}_{R}(\mathbf{X})$ is a Petri set. We consider a Presburger set $\mathbf{S} \subseteq \mathbb{Z}^{d}$. Observe that $\mathbf{X} \times \mathbf{S}$ is a Presburger relation. Since $R$ is a Petri relation we deduce that $R \cap(\mathbf{X} \times \mathbf{S})$ is 
a Lambert relation. Hence this set can be decomposed into a finite union $\bigcup_{j=1}^{k}\left(\mathbf{a}_{j}, \mathbf{b}_{j}\right)+R_{j}$ with $k \in \mathbb{N},\left(\mathbf{a}_{j}, \mathbf{b}_{j}\right) \in \mathbb{Z}^{d} \times \mathbb{Z}^{d}$ and $R_{j}$ is a polytope periodic relation. We deduce that $\operatorname{post}_{R}(\mathbf{X}) \cap \mathbf{S}=$ $\bigcup_{j=1}^{k} \mathbf{b}_{j}+\mathbf{P}_{j}$ where $\mathbf{P}_{j}=\left\{\mathbf{v} \in \mathbb{Z}^{d} \mid \exists(\mathbf{u}, \mathbf{v}) \in R_{j}\right\}$. Since $R_{j}$ is a periodic relation we deduce that $\mathbf{P}_{j}$ is a periodic set. Moreover since $\mathbb{Q} \geq 0 R_{j}$ is polytope we deduce that $\mathbf{C}_{j}=\left\{\mathbf{v} \in \mathbb{Q}^{d} \mid\right.$ $\left.\exists(\mathbf{u}, \mathbf{v}) \in \mathbb{Q}_{\geq 0} R_{j}\right\}$ is polytope. Let us prove that $\mathbb{Q}_{\geq 0} \mathbf{P}_{j}=\mathbf{C}_{j}$. By construction we have $\mathbf{P}_{j} \subseteq \mathbf{C}_{j}$. Since $\mathbf{C}_{j}$ is conic we deduce that $\mathbb{Q} \geq 0 \mathbf{P}_{j} \subseteq \mathbf{C}_{j}$. For the converse inclusion let $\mathbf{v} \in \mathbf{C}_{j}$. There exists $\mathbf{u} \in \mathbb{Q}^{d}$ such that $(\mathbf{u}, \mathbf{v}) \in \mathbb{Q}_{\geq 0} R_{j}$. Hence there exists $\lambda \in \mathbb{Q}>0$ such that $(\mathbf{u}, \mathbf{v}) \in \lambda \mathbf{R}_{j}$. Let us consider $n \in \mathbb{N}_{>0}$ such that $n \lambda_{j} \in \mathbb{N}$ and observe that $(n \mathbf{u}, n \mathbf{v}) \in(n \lambda) R_{j} \subseteq R_{j}$ since $R_{j}$ is periodic. Thus $n \mathbf{v} \in \mathbf{P}_{j}$ and we have proved that $\mathbf{v} \in \mathbb{Q}_{\geq 0} \mathbf{P}_{j}$. Hence $\mathbb{Q}_{\geq 0} \mathbf{P}_{j}=\mathbf{C}_{j}$ is a polytope conic set and we have proved that $\operatorname{post}_{R}(\mathbf{X}) \cap \mathbf{S}$ is a Lambert set for every Presburger set $\mathbf{S} \subseteq \mathbb{Z}^{d}$. Hence $\operatorname{post}_{R}(\mathbf{X})$ is a Petri set. From $\operatorname{pre}_{R}(\mathbf{Y})=\operatorname{post}_{R^{-1}}(\mathbf{Y})$ with $R^{-1}=\{(\mathbf{y}, \mathbf{x}) \mid(\mathbf{x}, \mathbf{y}) \in R\}$ we deduce that $\operatorname{pre}_{R}(\mathbf{Y})$ is a Petri set.

Now, let us prove Theorem 6.1. We consider a reflexive and transitive Petri relation $R^{*}$. We introduce the notion of separators. A separator is a couple $(\mathbf{X}, \mathbf{Y})$ of Presburger sets such that the intersection $R^{*} \cap(\mathbf{X} \times \mathbf{Y})$ is empty. Since $R^{*}$ is reflexive observe that $\mathbf{X} \cap \mathbf{Y}$ is empty. The Presburger set $\mathbf{D}=\mathbb{Z}^{d} \backslash(\mathbf{X} \cup \mathbf{Y})$ is called the domain of $(\mathbf{X}, \mathbf{Y})$. Let us observe that a separator $(\mathbf{X}, \mathbf{Y})$ with an empty domain is a partition of $\mathbb{Z}^{d}$ such that $\mathbf{X}$ is a Presburger forward invariant and $\mathbf{Y}$ is a Presburger backward invariant. In particular Theorem 6.1 is obtained thanks to the following Lemma 6.3 with an immediate induction.

Lemma 6.3. Let $\left(\mathbf{X}_{0}, \mathbf{Y}_{0}\right)$ be a separator with a non-empty domain $\mathbf{D}_{0}$. There exists a separator $(\mathbf{X}, \mathbf{Y})$ with a domain $\mathbf{D}$ such that $\mathbf{X}_{0} \subseteq \mathbf{X}, \mathbf{Y}_{0} \subseteq \mathbf{Y}$ and $\operatorname{dim}(\mathbf{D})<\operatorname{dim}\left(\mathbf{D}_{0}\right)$.

Proof. We first observe that a couple $(\mathbf{X}, \mathbf{Y})$ of Presburger sets is a separator if and only if $\operatorname{post}_{R^{*}}(\mathbf{X}) \cap \operatorname{pre}_{R^{*}}(\mathbf{Y})=\emptyset$ if and only if $\operatorname{post}_{R^{*}}(\mathbf{X}) \cap \mathbf{Y}=\emptyset$ if and only if $\operatorname{pre}_{R^{*}}(\mathbf{Y}) \cap \mathbf{X}=\emptyset$.

Since $R^{*}$ is a Petri relation we deduce that $\operatorname{post}_{R^{*}}\left(\mathbf{X}_{0}\right)$ is a Petri set. As $\mathbf{D}_{0}$ is a Presburger set, we deduce that post $R^{*}\left(\mathbf{X}_{0}\right) \cap$ $\mathbf{D}_{0}$ is a Lambert set. Hence post $R^{*}\left(\mathbf{X}_{0}\right) \cap \mathbf{D}_{0}=\bigcup_{j=1}^{k} \mathbf{b}_{j}+\mathbf{P}_{j}$ where $\mathbf{b}_{j} \in \mathbb{Z}^{d}$ and $\mathbf{P}_{j} \subseteq \mathbb{Z}^{d}$ is a polytope periodic set. We introduce the following Presburger set:

$$
\mathbf{S}=\bigcup_{j=1}^{k} \mathbf{b}_{j}+\operatorname{lin}\left(\mathbf{P}_{j}\right)
$$

Observe that post $R_{R^{*}}\left(\mathbf{X}_{0}\right) \cap \mathbf{D}_{0} \subseteq \mathbf{S}$. We deduce that the set $\mathbf{Y}=\mathbf{Y}_{0} \cup\left(\mathbf{D}_{0} \backslash \mathbf{S}\right)$ is such that post $R^{*}\left(\mathbf{X}_{0}\right) \cap \mathbf{Y}=\emptyset$. Hence $\left(\mathbf{X}_{0}, \mathbf{Y}\right)$ is a separator.

Symmetrically, since $R^{*}$ is a Petri relation we deduce that pre $_{R^{*}}(\mathbf{Y})$ is a Petri set. As $\mathbf{D}_{0}$ is a Presburger set, we deduce that $\operatorname{pre}_{R^{*}}(\mathbf{Y}) \cap \mathbf{D}_{0}$ is a Lambert set. Hence pre $R_{R^{*}}(\mathbf{Y}) \cap \mathbf{D}_{0}=$ $\bigcup_{l=1}^{n} \mathbf{c}_{l}+\mathbf{Q}_{l}$ where $\mathbf{c}_{l} \in \mathbb{Z}^{d}$ and $\mathbf{Q}_{l} \subseteq \mathbb{Z}^{d}$ is a polytope periodic set. We introduce the following Presburger set:

$$
\mathbf{T}=\bigcup_{l=1}^{n} \mathbf{c}_{l}+\operatorname{lin}\left(\mathbf{Q}_{l}\right)
$$

Observe that $\operatorname{pre}_{R^{*}}(\mathbf{Y}) \cap \mathbf{D}_{0} \subseteq \mathbf{T}$. We deduce that the set $\mathbf{X}=\mathbf{X}_{0} \cup\left(\mathbf{D}_{0} \backslash \mathbf{T}\right)$ is such that $\operatorname{pre}_{R^{*}}(\mathbf{Y}) \cap \mathbf{X}=\emptyset$. Hence $(\mathbf{X}, \mathbf{Y})$ is a separator.
Let us introduce the domain $\mathbf{D}$ of $(\mathbf{X}, \mathbf{Y})$. We have the following equality where $\mathbf{Z}_{j, l}=\left(\mathbf{b}_{j}+\operatorname{lin}\left(\mathbf{P}_{j}\right)\right) \cap\left(\mathbf{c}_{l}+\operatorname{lin}\left(\mathbf{Q}_{l}\right)\right)$ :

$$
\mathbf{D}=\mathbf{D}_{0} \cap\left(\bigcup_{\substack{1 \leq j \leq k \\ 1 \leq l \leq n}} \mathbf{Z}_{j, l}\right)
$$

As $(\mathbf{X}, \mathbf{Y})$ is a separator we deduce that $\operatorname{post}_{R^{*}}(\mathbf{X}) \cap \operatorname{pre}_{R^{*}}(\mathbf{Y})$ is empty. As $\mathbf{b}_{j}+\mathbf{P}_{j} \subseteq$ post $_{R^{*}}\left(\mathbf{X}_{0}\right) \subseteq$ post $_{R^{*}}(\mathbf{X})$ and $\mathbf{c}_{l}+\mathbf{Q}_{l} \subseteq$ $\operatorname{pre}_{R^{*}}(\mathbf{Y})$ we deduce that the intersection $\left(\mathbf{b}_{j}+\mathbf{P}_{j}\right) \cap\left(\mathbf{c}_{l}+\mathbf{Q}_{l}\right)$ is empty. Theorem 5.2 shows that $\operatorname{dim}\left(\mathbf{Z}_{j, l}\right)<\max \left\{\operatorname{dim}\left(\mathbf{b}_{j}+\right.\right.$ $\left.\left.\mathbf{P}_{j}\right), \operatorname{dim}\left(\mathbf{c}_{l}+\mathbf{Q}_{l}\right)\right\}$. Since $\mathbf{b}_{j}+\mathbf{P}_{j} \subseteq \mathbf{D}_{0}$ and $\mathbf{c}_{l}+\mathbf{Q}_{l} \subseteq \mathbf{D}_{0}$ we deduce that $\operatorname{dim}\left(\mathbf{b}_{j}+\mathbf{P}_{j}\right) \leq \operatorname{dim}\left(\mathbf{D}_{0}\right)$ and $\operatorname{dim}\left(\mathbf{c}_{l}+\overline{\mathbf{Q}}_{l}\right) \leq$ $\operatorname{dim}\left(\mathbf{D}_{0}\right)$. We have proved that $\operatorname{dim}(\mathbf{D})<\operatorname{dim}\left(\mathbf{D}_{0}\right)$.

\section{Vector Addition Systems}

In this section we introduce the Vector Addition Systems, the production relations and a well order over the set of runs of Vector Addition Systems.

A Vector Addition System (VAS) is a finite subset $\mathbf{A} \subseteq \mathbb{Z}^{d}$. A marking is a vector $\mathbf{m} \in \mathbb{N}^{d}$. The semantics of vector addition systems is obtained by introducing for every word $w=\mathbf{a}_{1} \ldots \mathbf{a}_{k}$ of vectors $\mathbf{a}_{j} \in \mathbf{A}$ the relation $\stackrel{w}{\longrightarrow}$ over the set of markings defined by $\mathbf{x} \stackrel{w}{\longrightarrow} \mathbf{y}$ if there exists a word $\rho=\mathbf{m}_{0} \ldots \mathbf{m}_{k}$ of markings $\mathbf{m}_{j} \in \mathbb{N}^{d}$ such that $(\mathbf{x}, \mathbf{y})=\left(\mathbf{m}_{0}, \mathbf{m}_{k}\right)$ and $\mathbf{m}_{j}=\mathbf{m}_{j-1}+\mathbf{a}_{j}$ for every $j \in\{1, \ldots, k\}$. The word $\rho$ is unique and it is called the run from $\mathbf{x}$ to $\mathbf{y}$ labeled by $w$. The marking $\mathbf{x}$ is called the source of $\rho$ and it is denoted by $\operatorname{src}(\rho)$, and the marking $\mathbf{y}$ is called the target of $\rho$ and it is denoted by $\operatorname{tgt}(\rho)$. The set of runs is denoted by $\Omega$.

The reachability relation is the relation denoted by $\stackrel{*}{\rightarrow}$ over the set of markings defined by $\mathbf{x} \stackrel{*}{\rightarrow} \mathbf{y}$ if there exists a word $w \in \mathbf{A}^{*}$ such that $\mathbf{x} \stackrel{w}{\longrightarrow} \mathbf{y}$. In the sequel we often used the fact that $\mathbf{x} \stackrel{w}{\longrightarrow} \mathbf{y}$ implies $\mathbf{x}+\mathbf{v} \stackrel{w}{\longrightarrow} \mathbf{y}+\mathbf{v}$ for every $\mathbf{v} \in \mathbb{N}^{d}$.

The production relation of a marking $\mathbf{m} \in \mathbb{N}^{d}$ is the relation $\stackrel{*}{\rightarrow} \mathbf{m}$ over $\mathbb{N}^{d}$ defined by $\mathbf{r} \stackrel{*}{\rightarrow} \mathbf{m}$ s if $\mathbf{m}+\mathbf{r} \stackrel{*}{\rightarrow} \mathbf{m}+\mathbf{s}$. The production relation of a run $\rho=\mathbf{m}_{0} \ldots \mathbf{m}_{k}$ is the relation $\stackrel{*}{\rightarrow} \rho$ defined by the following composition:

$$
\stackrel{*}{\rightarrow} \rho=\stackrel{*}{\rightarrow} \mathbf{m}_{0} \circ \cdots \circ \stackrel{*}{\rightarrow} \mathbf{m}_{k}
$$

The following Lemma 7.1 shows that $\stackrel{*}{\rightarrow} \rho$ is periodic for every run $\rho$ as a composition of periodic relations. Note that in Section 8 we prove that this periodic relation is polytope.

Lemma 7.1. The relation $\stackrel{*}{\rightarrow}_{\mathbf{m}}$ is periodic.

Proof. Let us assume that $\mathbf{r}_{1} \stackrel{*}{\rightarrow} \mathbf{m} \mathbf{s}_{1}$ and $\mathbf{r}_{2} \stackrel{*}{\rightarrow} \mathbf{m} \mathbf{s}_{2}$. Since $\mathbf{r}_{1} \stackrel{*}{\rightarrow} \mathbf{m} \mathbf{s}_{1}$ we deduce that $\mathbf{r}_{1}+\mathbf{r}_{2} \stackrel{*}{\rightarrow} \mathbf{m} \mathbf{s}_{1}+\mathbf{r}_{2}$. Moreover, since $\mathbf{r}_{2} \stackrel{*}{\rightarrow} \mathbf{m} \mathbf{s}_{2}$ we deduce that $\mathbf{r}_{2}+\mathbf{s}_{1} \stackrel{*}{\rightarrow} \mathbf{m} \mathbf{s}_{2}+\mathbf{s}_{1}$. Therefore $\mathbf{r}_{1}+\mathbf{r}_{2} \stackrel{*}{\rightarrow} \mathbf{m} \mathbf{s}_{1}+\mathbf{s}_{2}$.

We introduce a well order over the set of runs based on the following Lemma 7.2

Lemma 7.2. The following inclusion holds for every run $\rho$ :

$$
(\operatorname{src}(\rho), \operatorname{tgt}(\rho))+\stackrel{*}{\rightarrow} \rho \subseteq \stackrel{*}{\rightarrow}
$$

Proof. Assume that $\rho=\mathbf{m}_{0} \ldots \mathbf{m}_{k}$ with $\mathbf{m}_{j} \in \mathbb{N}^{d}$, and let $(\mathbf{r}, \mathbf{s})$ be a couple in the production relation $\stackrel{*}{\rightarrow}_{\rho}$. Since this relation is defined as a composition, there exists a sequence $\left(\mathbf{v}_{j}\right)_{0 \leq j \leq k+1}$ of vectors $\mathbf{v}_{j} \in \mathbb{N}^{d}$ satisfying the following relations with $\mathbf{v}_{0}=\mathbf{r}$ and $\mathbf{v}_{k+1}=\mathbf{s}$ :

$$
\mathbf{v}_{0} \stackrel{*}{\rightarrow} \mathbf{m}_{0} \mathbf{v}_{1} \cdots \mathbf{v}_{k} \stackrel{*}{\rightarrow} \mathbf{m}_{k} \mathbf{v}_{k+1}
$$


We introduce the vector $\mathbf{a}_{j}=\mathbf{m}_{j}-\mathbf{m}_{j-1}$ for every $j \in$ $\{1, \ldots, k\}$. Since $\mathbf{m}_{j-1} \stackrel{\mathbf{a}_{j}}{\longrightarrow} \mathbf{m}_{j}$ we deduce that $\mathbf{m}_{j-1}+\mathbf{v}_{j} \stackrel{\mathbf{a}_{j}}{\longrightarrow}$ $\mathbf{m}_{j}+\mathbf{v}_{j}$. Moreover, as $\mathbf{v}_{j} \stackrel{*}{\rightarrow} \mathbf{m}_{j} \mathbf{v}_{j+1}$, there exists a word $w_{j} \in \mathbf{A}^{*}$ such that $\mathbf{m}_{j}+\mathbf{v}_{j} \stackrel{w_{j}}{\longrightarrow} \mathbf{m}_{j}+\mathbf{v}_{j+1}$. We deduce that the following relation holds:

$$
\mathbf{m}_{0}+\mathbf{v}_{0} \stackrel{w_{0} \mathbf{a}_{1} w_{1} \ldots \mathbf{a}_{k} w_{k}}{\longrightarrow} \mathbf{m}_{k}+\mathbf{v}_{k+1}
$$

Therefore $\left(\mathbf{m}_{0}, \mathbf{m}_{k}\right)+\left(\mathbf{v}_{0}, \mathbf{v}_{k+1}\right)$ is in the reachability relation.

We introduce the order $\preceq$ over the set of runs defined by $\rho \preceq \rho^{\prime}$ if the following inclusion holds:

$$
\left(\operatorname{src}\left(\rho^{\prime}\right), \operatorname{tgt}\left(\rho^{\prime}\right)\right)+\stackrel{*}{\rightarrow} \rho^{\prime} \subseteq(\operatorname{src}(\rho), \operatorname{tgt}(\rho))+\stackrel{*}{\rightarrow} \rho
$$

In the reminder of this section we prove the following theorem. All other results or definitions introduced in this section are not used in the sequel.

Theorem 7.3. The order $\preceq$ is well.

The order $\preceq$ is proved well thanks to the Higmann's Lemma. We first recall this lemma. Let us consider an order $\sqsubseteq$ over a set $S$. We introduce the order $\complement^{*}$ over the set of words over $S$ defined by $u \sqsubseteq^{*} v$ where $u=s_{1} \ldots s_{k}$ with $s_{j} \in S$ if there exists a sequence $\left(t_{j}\right)_{1 \leq j \leq k}$ with $t_{j} \in S$ and $s_{j} \sqsubseteq t_{j}$ and a sequence $\left(w_{j}\right)_{0 \leq j \leq k}$ of words $w_{j} \in S^{*}$ such that $v=\bar{w}_{0} t_{1} w_{1} \ldots t_{k} w_{k}$.

Lemma 7.4 (Higmann's Lemma). The ordered set $\left(S^{*}, \sqsubseteq^{*}\right)$ is well for every well ordered set $(S, \sqsubseteq)$.

We associate to every run $\rho=\mathbf{m}_{0} \ldots \mathbf{m}_{k}$ the word $\alpha(\rho)=$ $\left(\mathbf{a}_{1}, \mathbf{m}_{1}\right) \ldots\left(\mathbf{a}_{k}, \mathbf{m}_{k}\right)$ where $\mathbf{a}_{j}=\mathbf{m}_{j}-\mathbf{m}_{j-1}$. Note that $\alpha(\rho)$ is a word over the alphabet $S=\mathbf{A} \times \mathbb{N}^{d}$. We introduce the order $\sqsubseteq$ over this alphabet by $(\mathbf{a}, \mathbf{m}) \sqsubseteq\left(\mathbf{a}^{\prime}, \mathbf{m}^{\prime}\right)$ if $\mathbf{a}=\mathbf{a}^{\prime}$ and $\mathbf{m} \leq \mathbf{m}^{\prime}$. Since $\mathbf{A}$ is a finite set and $\leq$ is a well order over $\mathbb{N}^{d}$, we deduce that $\sqsubseteq$ is a well order over $S$. From the Higmann's lemma, the order $\Xi^{*}$ is well over $S^{*}$. We introduce the well order $\unlhd$ over the set of runs defined by $\rho \unlhd \rho^{\prime}$ if $\alpha(\rho) \sqsubseteq^{*} \alpha\left(\rho^{\prime}\right), \operatorname{src}(\rho) \leq \operatorname{src}\left(\rho^{\prime}\right)$ and $\operatorname{tgt}(\rho) \leq \operatorname{tgt}\left(\rho^{\prime}\right)$. The following lemma provides a useful characterization of this order.

Lemma 7.5. Let $\rho=\mathbf{m}_{0} \ldots \mathbf{m}_{k}$ be a run and let $\rho^{\prime}$ be another run. We have $\rho \unlhd \rho^{\prime}$ if and only if there exists a sequence $\left(\mathbf{v}_{j}\right)_{0 \leq j \leq k+1}$ of vectors in $\mathbb{N}^{d}$ such that $\rho^{\prime}=\rho_{0}^{\prime} \ldots \rho_{k}^{\prime}$ where $\rho_{j}^{\prime}$ is a run from $\mathbf{m}_{j}+\mathbf{v}_{j}$ to $\mathbf{m}_{j}+\mathbf{v}_{j+1}$.

Proof. We introduce the sequence $\left(\mathbf{a}_{j}\right)_{1 \leq j \leq k}$ defined by $\mathbf{a}_{j}=$ $\mathbf{m}_{j}-\mathbf{m}_{j-1}$.

Assume first that $\rho \unlhd \rho^{\prime}$. Since $\alpha(\rho) \sqsubseteq^{*} \alpha\left(\rho^{\prime}\right)$ we deduce that $\alpha\left(\rho^{\prime}\right)=w_{0}\left(\mathbf{a}_{1}, \mathbf{m}_{1}^{\prime}\right) w_{1} \ldots\left(\mathbf{a}_{k}, \mathbf{m}_{k}^{\prime}\right) w_{k}$ where $w_{j} \in S^{*}$ and $\mathbf{m}_{j}^{\prime} \geq \mathbf{m}_{j}$. We introduce the sequence $\left(\mathbf{v}_{j}\right)_{0 \leq j \leq k+1}$ defined by $\mathbf{v}_{0}=\operatorname{src}\left(\rho^{\prime}\right)-\operatorname{src}(\rho), \mathbf{v}_{k+1}=\operatorname{tgt}\left(\rho^{\prime}\right)-\operatorname{tgt}(\rho)$ and $\mathbf{v}_{j}=$ $\mathbf{m}_{j}^{\prime}-\mathbf{m}_{j}$ for every $j \in\{1, \ldots, k\}$. Observe that $\mathbf{v}_{j} \in \mathbb{N}^{d}$ for every $j \in\{0, \ldots, k+1\}$. We deduce that $\rho^{\prime}$ can be decomposed into $\rho^{\prime}=\rho_{0}^{\prime} \ldots \rho_{k}^{\prime}$ where $\rho_{j}^{\prime}$ is the run from $\mathbf{m}_{j}+\mathbf{v}_{j}$ to $\mathbf{m}_{j}+\mathbf{v}_{j+1}$ such that $\alpha\left(\rho_{j}^{\prime}\right)=w_{j}$.

Conversely let $\left(\mathbf{v}_{j}\right)_{0 \leq j \leq k+1}$ be a sequence of vectors in $\mathbb{N}^{d}$ such that $\rho^{\prime}=\rho_{0}^{\prime} \ldots \rho_{k}^{\prime}$ where $\rho_{j}^{\prime}$ is a run from $\mathbf{m}_{j}+\mathbf{v}_{j}$ to $\mathbf{m}_{j}+\mathbf{v}_{j+1}$. We deduce that we have the following equality where $\mathbf{m}_{j}^{\prime}=\mathbf{m}_{j}+\mathbf{v}_{j}$ and $\mathbf{a}_{j}^{\prime} \in \mathbf{A}$ :

$$
\alpha\left(\rho^{\prime}\right)=\alpha\left(\rho_{0}^{\prime}\right)\left(\mathbf{a}_{1}^{\prime}, \mathbf{m}_{1}^{\prime}\right) \alpha\left(\rho_{1}^{\prime}\right) \ldots\left(\mathbf{a}_{k}^{\prime}, \mathbf{m}_{k}^{\prime}\right) \alpha\left(\rho_{k}^{\prime}\right)
$$

Observe that $\mathbf{a}_{j}^{\prime}=\operatorname{tgt}\left(\rho_{j-1}^{\prime}\right)-\mathbf{m}_{j}^{\prime}=\left(\mathbf{m}_{j}+\mathbf{v}_{j}\right)-\left(\mathbf{m}_{j-1}+\mathbf{v}_{j}\right)$ and in particular $\mathbf{a}_{j}^{\prime}=\mathbf{a}_{j}$. We deduce that $\alpha(\rho) \sqsubseteq^{*} \alpha\left(\rho^{\prime}\right)$.
Moreover, $\operatorname{since} \operatorname{src}(\rho) \leq \operatorname{src}\left(\rho^{\prime}\right)$ and $\operatorname{tgt}(\rho) \leq \operatorname{tgt}\left(\rho^{\prime}\right)$ we deduce that $\rho \unlhd \rho^{\prime}$.

Since $\unlhd$ is a well order, the following lemma shows that $\preceq$ is a well order. We have proved Theorem 7.3.

Lemma 7.6. $\rho \unlhd \rho^{\prime}$ implies $\rho \preceq \rho^{\prime}$.

Proof. Assume that $\rho=\mathbf{m}_{0} \ldots \mathbf{m}_{k}$. Lemma 7.5 shows that there exists a sequence $\left(\mathbf{v}_{j}\right)_{0 \leq j \leq k+1}$ of vectors in $\mathbb{N}^{d}$ such that $\rho^{\prime}=\rho_{0}^{\prime} \ldots \rho_{k}^{\prime}$ where $\rho_{j}^{\prime}$ is a run from $\mathbf{m}_{j}+\mathbf{v}_{j}$ to $\mathbf{m}_{j}+\mathbf{v}_{j+1}$. Lemma 7.2 shows that $\left(\operatorname{src}\left(\rho_{j}^{\prime}\right), \operatorname{tgt}\left(\rho_{j}^{\prime}\right)\right)+\stackrel{*}{\rightarrow} \rho_{j}^{\prime} \subseteq \stackrel{*}{\rightarrow}$. Hence $\left(\mathbf{v}_{j}, \mathbf{v}_{j+1}\right)+\stackrel{*}{\rightarrow} \rho_{j}^{\prime} \subseteq \stackrel{*}{\rightarrow} \mathbf{m}_{j}$. We deduce that $\left(\mathbf{v}_{0}, \mathbf{v}_{k+1}\right)+\stackrel{*}{\rightarrow} \rho^{\prime} \subseteq \stackrel{*}{\rightarrow} \rho$ by composition. Since $\left(\operatorname{src}\left(\rho^{\prime}\right), \operatorname{tgt}\left(\rho^{\prime}\right)\right)=(\operatorname{src}(\rho), \operatorname{tgt}(\rho))+$ $\left(\mathbf{v}_{0}, \mathbf{v}_{k+1}\right)$ we get $\rho \preceq \rho^{\prime}$ from the previous inclusion.

\section{Polytope Production Relations}

In this section we prove that production relations are polytope (Theorem 8.1). All other results or definitions introduced in the section are not used in the sequel.

\section{Theorem 8.1. Production relations are polytope.}

The following lemma shows that polytope periodic relations are stable by composition. In particular it is sufficient to prove that production relations $\stackrel{*}{\rightarrow} \mathbf{m}$ are polytope for every marking $\mathbf{m} \in \mathbb{N}^{d}$ in order to deduce that production relations $\stackrel{*}{\rightarrow} \rho_{\rho}$ are polytope for every run $\rho$.

Lemma 8.2. We have $\mathbb{Q} \geq 0\left(R_{1} \circ R_{2}\right)=\left(\mathbb{Q}_{\geq 0} R_{1}\right) \circ\left(\mathbb{Q}_{\geq 0} R_{2}\right)$ for every periodic relations over $\mathbb{Z}^{d}$.

Proof. We have $R_{1} \subseteq \mathbb{Q}_{\geq 0} R_{1}$ and $R_{2} \subseteq \mathbb{Q}_{\geq 0} R_{2}$. Thus $R_{1} \circ R_{2} \subseteq$ $C$ where $C=\left(\mathbb{Q} \geq 0 R_{1}\right) \circ\left(\mathbb{Q}_{\geq 0} R_{2}\right)$. As $\bar{C}$ is a conic set we get $\mathbb{Q}_{\geq 0}\left(R_{1} \circ R_{2}\right) \subseteq C$. For the converse inclusion, let us consider $(\mathbf{x}, \mathbf{z}) \in C$. There exists $\mathbf{y} \in \mathbb{Q}^{d}$ such that $(\mathbf{x}, \mathbf{y}) \in \mathbb{Q}_{\geq 0} R_{1}$ and $(\mathbf{y}, \mathbf{z}) \in \mathbb{Q}_{\geq 0} R_{2}$. There exists $\lambda_{1}, \lambda_{2} \in \mathbb{Q}_{\geq 0}$ such that $(\mathbf{x}, \mathbf{y}) \in$ $\lambda_{1} R_{1}$ and $(\mathbf{y}, \mathbf{z}) \in \lambda_{2} R_{2}$. We introduce $n_{1}, n_{2} \in \mathbb{N}_{>0}$ such that $n_{1} \lambda_{1} \in \mathbb{N}$ and $n_{2} \lambda_{2} \in \mathbb{N}$ and we deduce that $n(\mathbf{x}, \mathbf{y}) \in R_{1}$ and $n(\mathbf{y}, \mathbf{z}) \in R_{2}$ with $n=n_{1} n_{2}$. Hence $n(\mathbf{x}, \mathbf{z}) \in R_{1} \circ R_{2}$. We deduce that $(\mathbf{x}, \mathbf{z}) \in \mathbb{Q}_{\geq 0}\left(R_{1} \circ R_{2}\right)$.

Theorem 3.5 shows that the conic set $\mathbb{Q}_{\geq 0} \stackrel{*}{\rightarrow}$ m is polytope if and only if the following conic set is finitely generated for every vector space $V \subseteq \mathbb{Q}^{d} \times \mathbb{Q}^{d}$ :

$$
\overline{(\mathbb{Q} \geq 0 \stackrel{*}{\rightarrow} \mathbf{m}) \cap V}
$$

We introduce the periodic relation $\stackrel{*}{\rightarrow}_{\mathbf{m}, V}$ defined as the intersection $\stackrel{*}{\rightarrow}_{\mathbf{m}} \cap V$. Let us observe that $\left(\mathbb{Q}_{\geq 0} \stackrel{*}{\rightarrow}_{\mathbf{m}}\right) \cap V$ is equal to $\mathbb{Q} \geq 0 \stackrel{*}{\rightarrow} \mathbf{m}, V$. So, we just have to prove that the conic set $\overline{\mathbb{Q}_{\geq 0} \stackrel{*}{\rightarrow}} \mathbf{m}, V$ is finitely generated for every $\mathbf{m} \in \mathbb{N}^{d}$ and for every vector space $V \subseteq \mathbb{Q}^{d} \times \mathbb{Q}^{d}$.

We introduce the set $\Omega_{\mathbf{m}, V}$ of runs $\rho$ such that $(\operatorname{src}(\rho), \operatorname{tgt}(\rho))-$ $(\mathbf{m}, \mathbf{m})$ is in $\left(\mathbb{N}^{d} \times \mathbb{N}^{d}\right) \cap V$. Note that a couple $(\mathbf{r}, \mathbf{s}) \in \mathbb{N}^{d} \times \mathbb{N}^{d}$ satisfies $\mathbf{r} \stackrel{*}{\rightarrow} \mathbf{m}, V$ s if and only if there exists a run $\rho \in \Omega_{\mathbf{m}, V}$ such that $\operatorname{src}(\rho)=\mathbf{m}+\mathbf{r}$ and $\operatorname{tgt}(\rho)=\mathbf{m}+\mathbf{s}$. We introduce the set $\mathbf{Q}_{\mathbf{m}, V}$ of markings $\mathbf{q}$ that occurs in at least one run $\rho \in \Omega_{\mathbf{m}, V}$. In general the set $\mathbf{Q}_{\mathbf{m}, V}$ is infinite. We consider the set $I_{\mathbf{m}, V}$ of $i \in\{1, \ldots, d\}$ such that $\left\{\mathbf{q}(i) \mid \mathbf{q} \in \mathbf{Q}_{\mathbf{m}, V}\right\}$ is infinite. We observe that if $i \in I_{\mathbf{m}, V}$ there exists a sequence of markings in $\mathbf{Q}_{\mathbf{m}, V}$ such that the $i$ th component is strictly increasing. We are going to prove that there exists a sequence of markings in 
$\mathbf{Q}_{\mathbf{m}, V}$ such that every component in $I_{\mathbf{m}, V}$ is strictly increasing. This property is proved by introducing the intraproductions. An intraproduction for $(\mathbf{m}, V)$ is a triple $(\mathbf{r}, \mathbf{x}, \mathbf{s})$ such that $\mathbf{x} \in \mathbb{N}^{d}$, $(\mathbf{r}, \mathbf{s}) \in\left(\mathbb{N}^{d} \times \mathbb{N}^{d}\right) \cap V$ and such that:

$$
\mathbf{r} \stackrel{*}{\rightarrow} \mathbf{m} \mathbf{x} \stackrel{*}{\rightarrow}_{\mathbf{m}} \mathbf{S}
$$

Since $\stackrel{*}{\rightarrow} \mathbf{m}$ is a periodic relation we deduce that the set of intraproductions is stable by addition. In particular $\mathbf{m}+n \mathbf{x}$ occurs in at least one run of $\Omega_{\mathbf{m}, V}$ for every intraproduction $(\mathbf{r}, \mathbf{x}, \mathbf{s})$ and for every $n \in \mathbb{N}$. Hence, if $\mathbf{x}(i)>0$ then $i \in I_{\mathbf{m}, V}$. An intraproduction for $(\mathbf{m}, V)$ is said to be total if $\mathbf{x}(i)>0$ for every $i \in I_{\mathbf{m}, V}$.

Lemma 8.3. There exists a total intraproduction for $(\mathbf{m}, V)$.

Proof. Since finite sums of intraproductions are intraproductions, it is sufficient to prove that for every $i \in I_{\mathbf{m}, V}$ there exists an intraproduction $(\mathbf{r}, \mathbf{x}, \mathbf{s})$ for $(\mathbf{m}, V)$ such that $\mathbf{x}(i)>0$. We fix $i \in I$.

Let us first prove that there exists $\mathbf{q} \leq \mathbf{q}^{\prime}$ in $\mathbf{Q}_{\mathbf{m}, V}$ such that $\mathbf{q}(i)<\mathbf{q}^{\prime}(i)$. Since $i \in I$ there exists a sequence $\left(\mathbf{q}_{n}\right)_{n \in \mathbb{N}}$ of markings $\mathbf{q}_{n} \in \mathbf{Q}_{\mathbf{m}, V}$ such that $\left(\mathbf{q}_{n}(i)\right)_{n \in \mathbb{N}}$ is strictly increasing. Since $\left(\mathbb{N}^{d}, \leq\right)$ is well ordered, we can extract for this sequence a subsequence that is non decreasing for $\leq$. We have proved that there exists $\mathbf{q} \leq \mathbf{q}^{\prime}$ in $\mathbf{Q}_{\mathbf{m}, V}$ such that $\mathbf{q}(i)<\mathbf{q}^{\prime}(i)$.

As $\mathbf{q} \in \mathbf{Q}_{\mathbf{m}, V}$ then $\mathbf{q}$ occurs in a run in $\Omega_{\mathbf{m}, V}$. Hence there exists $(\mathbf{r}, \mathbf{s}) \in\left(\mathbb{N}^{d} \times \mathbb{N}^{d}\right) \cap V$ such that:

$$
\mathbf{m}+\mathbf{r} \stackrel{*}{\rightarrow} \mathbf{q} \stackrel{*}{\rightarrow} \mathbf{m}+\mathbf{s}
$$

Symmetrically, as $\mathbf{q}^{\prime} \in \mathbf{Q}_{\mathbf{m}, V}$ there exists $\left(\mathbf{r}^{\prime}, \mathbf{s}^{\prime}\right) \in\left(\mathbb{N}^{d} \times \mathbb{N}^{d}\right) \cap V$ such that:

$$
\mathbf{m}+\mathbf{r}^{\prime} \stackrel{*}{\rightarrow} \mathbf{q}^{\prime} \stackrel{*}{\rightarrow} \mathbf{m}+\mathbf{s}^{\prime}
$$

Let us introduce $\mathbf{v}=\mathbf{q}^{\prime}-\mathbf{q}$. We deduce:

- $\left(\mathbf{m}+\mathbf{r}^{\prime}\right)+\mathbf{r} \stackrel{*}{\rightarrow} \mathbf{q}^{\prime}+\mathbf{r}$ from $\mathbf{m}+\mathbf{r}^{\prime} \stackrel{*}{\rightarrow} \mathbf{q}^{\prime}$.

- $\mathbf{q}+(\mathbf{v}+\mathbf{r}) \stackrel{*}{\rightarrow}(\mathbf{m}+\mathbf{s})+(\mathbf{v}+\mathbf{r})$ from $\mathbf{q} \stackrel{*}{\rightarrow} \mathbf{m}+\mathbf{s}$.

- $(\mathbf{m}+\mathbf{r})+(\mathbf{v}+\mathbf{s}) \stackrel{*}{\rightarrow} \mathbf{q}+(\mathbf{v}+\mathbf{s})$ from $\mathbf{m}+\mathbf{r} \stackrel{*}{\rightarrow} \mathbf{q}$.

- $\mathbf{q}^{\prime}+\mathbf{s} \stackrel{*}{\rightarrow}\left(\mathbf{m}+\mathbf{s}^{\prime}\right)+\mathbf{s}$ from $\mathbf{q}^{\prime} \stackrel{*}{\rightarrow} \mathbf{m}+\mathbf{s}^{\prime}$.

Since $\mathbf{q}^{\prime}+\mathbf{r}=\mathbf{q}+\mathbf{v}+\mathbf{r}$ and $\mathbf{q}+\mathbf{v}+\mathbf{s}=\mathbf{q}^{\prime}+\mathbf{s}$, we have proved the following relations where $\mathbf{x}=\mathbf{s}+\mathbf{v}+\mathbf{r}$ :

$$
\mathbf{r}+\mathbf{r}^{\prime} \stackrel{*}{\rightarrow} \mathbf{m} \mathbf{x} \stackrel{*}{\rightarrow} \mathbf{m} \mathbf{s}+\mathbf{s}^{\prime}
$$

As $\left(\mathbf{r}+\mathbf{r}^{\prime}, \mathbf{s}+\mathbf{s}^{\prime}\right) \in\left(\mathbb{N}^{d} \times \mathbb{N}^{d}\right) \cap V$ we deduce that $\left(\mathbf{r}+\mathbf{r}^{\prime}, \mathbf{x}, \mathbf{s}+\mathbf{s}^{\prime}\right)$ is an intraproduction for $(\mathbf{m}, V)$. Since $\mathbf{x}(i)>0$ we are done.

Let us introduce an additional element $\infty \notin \mathbb{N}$ and let $\mathbb{N}_{\infty}=$ $\mathbb{N} \cup\{\infty\}$. A vector in $\mathbb{N}_{\infty}^{d}$ is called an extended marking and the set $I=\{i \in\{1, \ldots, d\} \mid \mathbf{m}(i)=\infty\}$ is called the set of relaxed components of an extended marking $\mathbf{m}$. Given a finite set $I \subseteq\{1, \ldots, d\}$ and a marking $\mathbf{m} \in \mathbb{N}^{d}$, we denote by $\mathbf{m}^{I}$ the extended marking defined by $\mathbf{m}^{I}(i)=\infty$ if $i \in I$ and $\mathbf{m}^{I}(i)=\mathbf{m}(i)$ if $i \notin I$. Given a word $w=\mathbf{a}_{1} \ldots \mathbf{a}_{k}$ of vectors $\mathbf{a}_{j} \in \mathbf{A}$, we extend the relation $\stackrel{w}{\longrightarrow}$ over the set of extended markings relaxed over a set $I$ by $\mathbf{x} \stackrel{w}{\longrightarrow} \mathbf{y}$ if there exists a word $\rho=\mathbf{m}_{0} \ldots \mathbf{m}_{k}$ of extended markings relaxed over $I$ such that $(\mathbf{x}, \mathbf{y})=\left(\mathbf{m}_{0}, \mathbf{m}_{k}\right)$ and $\mathbf{m}_{j}(i)=\mathbf{m}_{j-1}(i)+\mathbf{a}_{j}(i)$ for every $j \in\{1, \ldots, k\}$ and for every $i \in\{1, \ldots, d\} \backslash I$. The word $\rho$ is unique and it is called the run from $\mathbf{x}$ to $\mathbf{y}$ labeled by $w$.

We introduce the finite graph $G_{\mathbf{m}, V}=(\mathbf{Q}, \mathbf{A}, E)$ where $\mathbf{Q}=$ $\left\{\mathbf{q}^{I_{\mathbf{m}, V}} \mid \mathbf{q} \in \mathbf{Q}_{\mathbf{m}, V}\right\}$ and where $E=\left\{\left(\mathbf{p}^{I_{\mathbf{m}, V}}, \mathbf{a}, \mathbf{q}^{I_{\mathbf{m}, V}}\right) \mid\right.$ $\left.\mathbf{p}, \mathbf{q} \in \mathbf{Q}_{\mathbf{m}, V} \wedge \mathbf{q}=\mathbf{p}+\mathbf{a}\right\}$. We introduce the periodic relation
$R_{\mathbf{m}, V}$ of couples $(\mathbf{r}, \mathbf{s}) \in\left(\mathbb{N}^{d} \times \mathbb{N}^{d}\right) \cap V$ such that $\mathbf{r}(i)=\mathbf{s}(i)=0$ for every $i \in\{1, \ldots, d\} \backslash I_{\mathbf{m}, V}$ and such that there exists a cycle in $G_{\mathbf{m}, V}$ on the state $\mathbf{m}^{I_{\mathbf{m}, V}}$ labeled by a word $\mathbf{a}_{1} \ldots \mathbf{a}_{k}$ where $\mathbf{a}_{j} \in \mathbf{A}$ such that $\mathbf{r}+\sum_{j=1}^{k} \mathbf{a}_{j}=\mathbf{s}$.

Lemma 8.4. The periodic relation $R_{\mathbf{m}, V}$ is Presburger.

Proof. This is a classical result based on the fact that the Parikh image of a regular language is Presburger.

Lemma 8.5. The following equality holds:

$$
\overline{\mathbb{Q} \geq 0} R_{\mathbf{m}, V}=\overline{\mathbb{Q}_{\geq 0} \stackrel{*}{\rightarrow} \mathbf{m}, V}
$$

Proof. Let us first prove the inclusion $\supseteq$. Let $(\mathbf{r}, \mathbf{s})$ such that $\mathbf{r} \stackrel{*}{\rightarrow} \mathbf{m}, V$ s. In this case there exists a word $w \in \mathbf{A}^{*}$ such that $\mathbf{m}+\mathbf{r} \stackrel{w}{\longrightarrow} \mathbf{m}+\mathbf{s}$. Observe that $\mathbf{m}+n \mathbf{r}$ and $\mathbf{m}+n \mathbf{s}$ are in $\mathbf{Q}_{\mathbf{m}, V}$ for every $n \in \mathbb{N}$. Hence $\mathbf{r}(i)>0$ or $\mathbf{s}(i)>0$ implies $i \in I_{\mathbf{m}, V}$ and we deduce that $\mathbf{m}^{I_{\mathbf{m}, V}} \stackrel{w}{\longrightarrow} \mathbf{m}^{I_{\mathbf{m}, V}}$. Therefore $w$ is the label of cycle in $G_{\mathbf{m}, V}$ on $\mathbf{m}^{I_{\mathbf{m}}, V}$. We have proved that $(\mathbf{r}, \mathbf{s}) \in R_{\mathbf{m}, V}$.

Now let us prove the inclusion $\subseteq$. We consider $(\mathbf{r}, \mathbf{s}) \in R_{\mathbf{m}, V}$. In this case $(\mathbf{r}, \mathbf{s}) \in\left(\mathbb{N}^{d} \times \mathbb{N}^{d}\right) \cap \bar{V}$ satisfies $\mathbf{r}(i)=\mathbf{s}(i)=0$ for every $i \notin I_{\mathbf{m}, V}$ and there exists a word $w=\mathbf{a}_{1} \ldots \mathbf{a}_{k}$ of vectors $\mathbf{a}_{j} \in \mathbf{A}$ that labels a cycle in $G_{\mathbf{m}, V}$ on $\mathbf{m}^{I_{\mathbf{m}, V}}$ and such that $\mathbf{m}+\mathbf{r}+\sum_{j=1}^{k} \mathbf{a}_{j}=\mathbf{m}+\mathbf{s}$. Let us consider a total intraproduction $\left(\mathbf{r}^{\prime}, \mathbf{x}, \mathbf{s}^{\prime}\right)$ for $(\mathbf{m}, V)$. Given $p \in \mathbb{N}$ and $j \in\{0, \ldots, k\}$ we introduce the following vector $\mathbf{m}_{p, j}$ :

$$
\mathbf{m}_{p, j}=\mathbf{m}+\mathbf{r}+p \mathbf{x}+\mathbf{a}_{1}+\cdots+\mathbf{a}_{j}
$$

Let us first prove that there exists $p \in \mathbb{N}$ such that $\mathbf{m}_{p, j}(i) \in \mathbb{N}$ for every $i \in I_{\mathbf{m}, V}$ and $j \in\{0, \ldots, k\}$. Let $i \in I_{\mathbf{m}, V}$ and $j \in\{0, \ldots, k\}$, since $\mathbf{x}(i)>0$, there exists $p_{i, j} \in \mathbb{N}$ such that $\mathbf{m}_{p, j}(i) \in \mathbb{N}$ for every $p \geq p_{i, j}$. We deduce that there exists $p \in \mathbb{N}$ such that $\mathbf{m}_{p, j}(i) \in \mathbb{N}$ for every $i \in I_{\mathbf{m}, V}$ and $j \in\{0, \ldots, k\}$.

Now we prove that $\mathbf{m}_{p, j}(i) \in \mathbb{N}$ for every $i \in\{1, \ldots, d\} \backslash I_{\mathbf{m}, V}$ and $j \in\{0, \ldots, k\}$. Let $j \in\{0, \ldots, k\}$. Since $w$ is the label of a cycle on $\mathbf{m}^{I_{\mathbf{m}, V}}$, there exists an extended marking $\mathbf{q}_{j}$ relaxed over $I_{\mathbf{m}, V}$ such that the following relation holds:

$$
\mathbf{m}^{I_{\mathbf{m}, V}} \stackrel{\mathbf{a}_{1} \ldots \mathbf{a}_{j}}{\longrightarrow} \mathbf{q}_{j}
$$

We deduce that for every $i \in\{1, \ldots, d\} \backslash I_{\mathbf{m}, V}$ we have $\mathbf{m}(i)+$ $\mathbf{a}_{1}(i)+\cdots+\mathbf{a}_{j}(i)=\mathbf{q}_{j}(i)$. Since $\mathbf{r}(i)=0$ and $\mathbf{x}(i)=0$ we get $\mathbf{m}_{p, j}(i) \in \mathbb{N}$.

We have proved that $\mathbf{m}_{p, j} \in \mathbb{N}^{d}$ for every $j \in\{0, \ldots, k\}$. Since $\mathbf{m}_{p, j}-\mathbf{m}_{p, j-1}=\mathbf{a}_{j}$ we deduce that $\rho_{p}=\mathbf{m}_{p, 0} \ldots \mathbf{m}_{p, k}$ is a run. Note that $\mathbf{m}_{p, 0}=\mathbf{m}+p \mathbf{x}+\mathbf{r}$ and $\mathbf{m}_{p, k}=\mathbf{m}+p \mathbf{x}+$ $\mathbf{r}+\sum_{j=1}^{k} \mathbf{a}_{j}=\mathbf{m}+p \mathbf{x}+\mathbf{s}$. We have proved that the following relation holds:

$$
\mathbf{m}+p \mathbf{x}+\mathbf{r} \stackrel{w}{\longrightarrow} \mathbf{m}+p \mathbf{x}+\mathbf{s}
$$

In particular $(\mathbf{r}, \mathbf{s})$ is in the production relation ${ }_{\rightarrow}^{*} \mathbf{m}^{\prime}$ where $\mathbf{m}^{\prime}=$ $\mathbf{m}+p \mathbf{x}$. Since a production relation is periodic we get $\mathbf{m}^{\prime}+n \mathbf{r} \stackrel{*}{\rightarrow}$ $\mathbf{m}^{\prime}+n \mathbf{s}$ for every $n \in \mathbb{N}$. As $\left(p \mathbf{r}^{\prime}, p \mathbf{x}, p \mathbf{s}^{\prime}\right)$ is an intraproduction for $(\mathbf{m}, V)$ we get $\mathbf{m}+p \mathbf{r}^{\prime} \stackrel{*}{\rightarrow} \mathbf{m}^{\prime} \stackrel{*}{\rightarrow} \mathbf{m}+p \mathbf{s}^{\prime}$. We deduce the relation $\left(\mathbf{m}+p \mathbf{r}^{\prime}\right)+n \mathbf{r} \stackrel{*}{\rightarrow} \mathbf{m}^{\prime}+n \mathbf{r}$ from $\left(\mathbf{m}+p \mathbf{r}^{\prime}\right) \stackrel{*}{\rightarrow} \mathbf{m}^{\prime}$, and the relation $\mathbf{m}^{\prime}+n \mathbf{s} \stackrel{*}{\rightarrow}\left(\mathbf{m}+p \mathbf{s}^{\prime}\right)+n \mathbf{s}$ from $\mathbf{m}^{\prime} \stackrel{*}{\rightarrow}\left(\mathbf{m}+p \mathbf{s}^{\prime}\right)$. We deduce that the following relation holds for every $n \in \mathbb{N}$ :

$$
\mathbf{m}+p \mathbf{r}^{\prime}+n \mathbf{r} \stackrel{*}{\rightarrow} \mathbf{m}+p \mathbf{s}^{\prime}+n \mathbf{s}
$$

Hence $p\left(\mathbf{r}^{\prime}, \mathbf{s}^{\prime}\right)+\mathbb{N}(\mathbf{r}, \mathbf{s}) \subseteq \stackrel{*}{\rightarrow} \mathbf{m}, V$. Thus $(\mathbf{r}, \mathbf{s}) \in \overline{\mathbb{Q}_{\geq 0} \stackrel{*}{\rightarrow} \mathbf{m}, V}$. From the inclusion $R_{\mathbf{m}, V} \subseteq \overline{\mathbb{Q}_{\geq 0} \stackrel{*}{\rightarrow} \mathbf{m}, V}$ we get the inclusion $\overline{\mathbb{Q} \geq 0} R_{\mathbf{m}, V} \subseteq \overline{\mathbb{Q}_{\geq 0} \stackrel{*}{\rightarrow} \mathbf{m}, V}$. 
Lemma 8.6. The conic set $\overline{\mathbb{Q}>0} \mathbf{P}$ if finitely generated for every Presburger periodic set $\mathbf{P}$.

Proof. Let us consider a Presburger periodic set $\mathbf{P}$. Since $\mathbf{P}$ is Presburger then $\mathbf{P}=\bigcup_{j=1}^{k} \mathbf{b}_{j}+\mathbf{P}_{j}$ where $\mathbf{b}_{j} \in \mathbb{Z}^{d}$ and $\mathbf{P}_{j} \subseteq$ $\mathbb{Z}^{d}$ is a finitely generated periodic set. We introduce the finitely generated conic set $\mathbf{C}=\sum_{j=1}^{k}\left(\mathbb{Q}_{\geq 0} \mathbf{b}_{j}+\mathbf{C}_{j}\right)$ where $\mathbf{C}_{j}$ is the finitely generated conic set $\mathbf{C}_{j}=\mathbb{Q}_{\geq 0} \mathbf{P}_{j}$. Since $\mathbf{P} \subseteq \mathbf{C}$ and $\mathbf{C}$ is a conic set we deduce the inclusion $\mathbb{Q}_{\geq 0} \mathbf{P} \subseteq \mathbf{C}$. As $\mathbf{C}$ is finitely generated we deduce that $\mathbf{C}$ is closed. Hence $\overline{\mathbb{Q}>0} \mathbf{P} \subseteq \mathbf{C}$. For the other inclusion let $\mathbf{p} \in \mathbf{P}_{j}$. For every $n \in \mathbb{N}$ we have $\mathbf{b}_{j}+n \mathbf{p} \in \mathbf{P}$. Hence $\frac{1}{n} \mathbf{b}_{j}+\mathbf{p} \in \mathbb{Q}_{\geq 0} \mathbf{P}$ for every $n \in \mathbb{N}_{>0}$. We deduce that $\mathbf{p} \in \overline{\mathbb{Q} \geq 0} \mathbf{P}$. Therefore $\mathbf{P}_{j} \subseteq \overline{\mathbb{Q} \geq 0} \mathbf{P}$. We deduce that $\mathbf{C}_{j} \subseteq \overline{\mathbb{Q}_{\geq 0} \mathbf{P}}$. As $\mathbb{Q}_{\geq 0} \mathbf{b}_{j} \subseteq \mathbb{Q}_{\geq 0} \mathbf{P} \subseteq \overline{\mathbb{Q}_{\geq 0} \mathbf{P}}$ we have proved the inclusion $\mathbf{C} \subseteq \overline{\mathbb{Q} \geq 0} \mathbf{P}$. Hence the previous inclusion is in fact an equality.

Now, we can prove Theorem 8.1. Lemma 8.4 shows that $R_{\mathbf{m}, V}$ is a Presburger periodic relation. Lemma 8.6 proves that the conic set $\overline{\mathbb{Q} \geq 0} R_{\mathbf{m}, V}$ is finitely generated. Lemma 8.5 shows

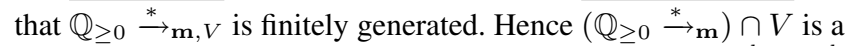
finitely generated conic set for every vector space $V \subseteq \mathbb{Q}^{d} \times \mathbb{Q}^{d}$. Theorem 3.5 shows that the conic relation $\mathbb{Q}_{\geq 0} \stackrel{*}{\rightarrow} \mathbf{m}$ is polytope. Hence $\stackrel{*}{\rightarrow} \mathbf{m}$ is a polytope periodic relation.

\section{Petri Reachability Relations}

In this section we prove the following Theorem 9.1. All other results or definitions introduced in this section are not used in the sequel.

Theorem 9.1. The reachability relation of a Vector Addition System is a Petri relation.

We are interested in proving that $\stackrel{*}{\rightarrow}$ is a Petri relation. This problem is equivalent to prove that $\stackrel{*}{\rightarrow} \cap((\mathbf{m}, \mathbf{n})+P)$ is a Lambert relation for every $(\mathbf{m}, \mathbf{n}) \in \mathbb{N}^{d} \times \mathbb{N}^{d}$ and for every finitely generated periodic relation $P \subseteq \mathbb{N}^{d} \times \mathbb{N}^{d}$. We introduce the order $\leq_{P}$ over $P$ defined by $p \leq_{P} \overline{p^{\prime}}$ if $p^{\prime} \in p+P$. Since $P$ is finitely generated we deduce that $\leq_{P}$ is a well order over $P$ (Dickson's Lemma). We introduce the set $\Omega_{\mathbf{m}, P, \mathbf{n}}$ of runs $\rho$ such that $(\operatorname{src}(\rho), \operatorname{tgt}(\rho)) \in$ $(\mathbf{m}, \mathbf{n})+P$. This set is well ordered by the relation $\preceq_{P}$ defined by $\rho \preceq_{P} \quad \rho^{\prime}$ if $\rho \preceq \rho^{\prime},(\operatorname{src}(\rho), \operatorname{tgt}(\rho))-(\mathbf{m}, \mathbf{n}) \leq_{P}$ $\left(\operatorname{src}\left(\rho^{\prime}\right), \operatorname{tgt}\left(\rho^{\prime}\right)\right)-(\mathbf{m}, \mathbf{n})$. We deduce that $\min _{\preceq_{P}}\left(\Omega_{\mathbf{m}, P, \mathbf{n}}\right)$ is finite.

Lemma 9.2. The following equality holds:

$$
\stackrel{*}{\rightarrow} \cap((\mathbf{m}, \mathbf{n})+P)=\underset{\rho \in \min _{\preceq}\left(\Omega_{\mathbf{m}, P, \mathbf{n}}\right)}{\bigcup_{(j)}}(\operatorname{src}(\rho), \operatorname{tgt}(\rho))+\left(\stackrel{*}{\rightarrow} \rho_{\rho} \cap P\right)
$$

Proof. Let us first prove $\supseteq$. Let $\rho \in \Omega_{\mathbf{m}, P, \mathbf{n}}$. Lemma 7.2 shows that $(\operatorname{src}(\rho), \operatorname{tgt}(\rho))+\stackrel{*}{\rightarrow} \rho \subseteq \stackrel{*}{\longrightarrow}$. Since $(\operatorname{src}(\rho), \operatorname{tgt}(\rho)) \in(\mathbf{m}, \mathbf{n})+P$ and $P$ is periodic we deduce the inclusion $\supseteq$.

Let us prove $\subseteq$. Let $\left(\mathbf{x}^{\prime}, \mathbf{y}^{\prime}\right)$ in the intersection $\stackrel{*}{\rightarrow} \cap((\mathbf{m}, \mathbf{n})+$ $P)$. There exists a run $\rho^{\prime} \in \Omega_{\mathbf{m}, P, \mathbf{n}}$ such that $\mathbf{x}^{\prime}=\operatorname{src}\left(\rho^{\prime}\right)$ and $\mathbf{y}^{\prime}=\operatorname{tgt}\left(\rho^{\prime}\right)$. Since $\preceq_{P}$ is a well order, there exists $\rho \in$ $\min _{\preceq_{P}}\left(\Omega_{\mathbf{m}, P, \mathbf{n}}\right)$ such that $\rho \preceq_{P} \rho^{\prime}$. We deduce that $\left(\mathbf{x}^{\prime}, \mathbf{y}^{\prime}\right)$ is in $(\operatorname{src}(\rho), \operatorname{tgt}(\rho))+\stackrel{*}{\rightarrow} \rho$. We get $\left(\mathbf{x}^{\prime}, \mathbf{y}^{\prime}\right) \in(\operatorname{src}(\rho), \operatorname{tgt}(\rho))+(\stackrel{*}{\rightarrow} \rho$ $\cap P)$ and we have proved the inclusion $\subseteq$.

Theorem 8.1 shows $\stackrel{*}{\rightarrow} \rho$ is a polytope periodic relation. Since $P$ is a finitely generated relation we deduce that $P$ is a polytope periodic relation. Lemma 4.5 shows that the class of polytope periodic relations is stable by finite intersections. We deduce that $\stackrel{*}{\rightarrow} \rho \cap P$ is polytope periodic relation. Thanks to the previous lemma we deduce that $\stackrel{*}{\rightarrow} \cap((\mathbf{m}, \mathbf{n})+P)$ is a Lambert relation for every $(\mathbf{m}, \mathbf{n}) \in \mathbb{N}^{d} \times \mathbb{N}^{d}$ and for every finitely generated periodic relation $P \subseteq \mathbb{N}^{d} \times \mathbb{N}^{d}$. Therefore $\stackrel{*}{\rightarrow}$ is a Petri relation and we have proved Theorem 9.1.

\section{Conclusion}

The reachability problem for Vector Additions Systems consists to decide for a triple $(\mathbf{m}, \mathbf{A}, \mathbf{n})$ where $\mathbf{m}, \mathbf{n}$ are two markings of a Vector Addition System $\mathbf{A}$ if there exists a word $w \in \mathbf{A}^{*}$ such that $\mathbf{m} \stackrel{w}{\longrightarrow} \mathbf{n}$. The following algorithm decides this problem.

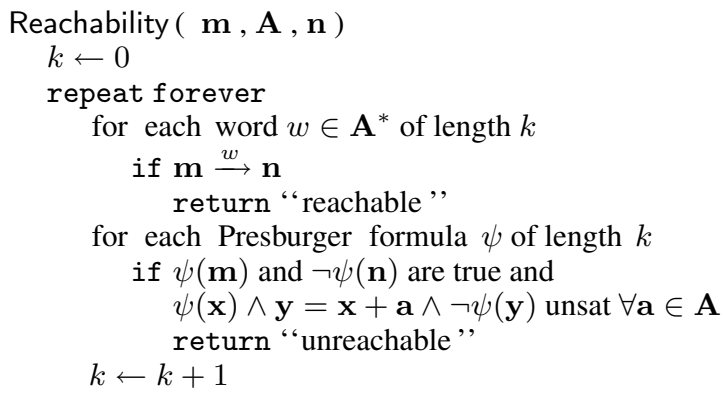

The correctness is immediate and the termination is guaranteed by the following Theorem 10.1.

Theorem 10.1. For every pair of markings $(\mathbf{m}, \mathbf{n})$ in the complement of the reachability relation of a Vector Addition System, there exists a partition of the set of markings into a Presburger forward invariant that contains $\mathbf{m}$ and a Presburger backward invariant that contains $\mathbf{n}$.

Proof. Let us consider $\mathbf{X}=\{\mathbf{m}\}$ and $\mathbf{Y}=\{\mathbf{n}\}$ and let $R^{*}$ be the reachability relation of the Vector addition system. Theorem 9.1 shows that $R^{*}$ is a Petri relation. Since $R^{*}$ is reflexive and transitive and such that $(\mathbf{X} \times \mathbf{Y}) \cap R^{*}=\emptyset$, Theorem 6.1 shows that there exists a partition of the set of markings into a Presburger forward invariant set that contains $\mathbf{X}$ and a Presburger backward invariant set that contains $\mathbf{Y}$.

This algorithm does not require the classical KLMST decomposition. Note however that the complexity of this algorithm is still open. In fact, the complexity depends on the minimal size of a word $w \in \mathbf{A}^{*}$ such that $\mathbf{m} \stackrel{w}{\longrightarrow} \mathbf{n}$ if $\mathbf{m} \stackrel{*}{\rightarrow} \mathbf{n}$, and the minimal size of a Presburger formula $\psi(\mathbf{x})$ denoting a forward invariant $\mathbf{M}$ such that $\mathbf{m} \in \mathbf{M}$ and $\mathbf{n} \notin \mathbf{M}$ otherwise. We left as an open question the problem of computing lower and upper bounds for these sizes. Note that the VAS exhibiting a large (Ackermann size) but finite reachability set given in [MM81] does not directly provide an Ackermann lower-bound for these sizes since Presburger forward invariants can over-approximate reachability sets.

As future work we are interested in providing complexity bounds on formulas in $\mathrm{FO}(\mathbb{Q},+, \leq, 0,1)$ denoting the polytope conic sets $\mathbb{Q}_{\geq 0} \stackrel{*}{\rightarrow} \mathbf{m}$.

\section{References}

[EN94] J. Esparza and M. Nielsen. Decidability issues for petri nets a survey. Bulletin of the European Association for Theoretical Computer Science, 52:245-262, 1994.

[GS66] Seymour Ginsburg and Edwin H. Spanier. Semigroups, Presburger formulas and languages. Pacific Journal of Mathematics, 16(2):285-296, 1966. 
[Hau90] Dirk Hauschildt. Semilinearity of the Reachability Set is Decidable for Petri Nets. PhD thesis, University of Hamburg, 1990.

[Kos82] S. Rao Kosaraju. Decidability of reachability in vector addition systems (preliminary version). In Proceedings of the Fourteenth Annual ACM Symposium on Theory of Computing, (STOC 1982), 5-7 May 1982, San Francisco, California, USA, pages 267-281. ACM, 1982.

[Lam92] Jean Luc Lambert. A structure to decide reachability in petri nets. Theoretical Computer Science, 99(1):79-104, 1992.

[Ler09] Jérôme Leroux. The general vector addition system reachability problem by Presburger inductive invariants. In LICS'09, pages 4-13, 2009.

[May81] Ernst W. Mayr. An algorithm for the general petri net reachability problem. In Conference Proceedings of the Thirteenth Annual ACM Symposium on Theory of Computation, (STOC 1981), 11-13 May 1981, Milwaukee, Wisconsin, USA, pages 238-246. ACM, 1981.

[MM81] Ernst W. Mayr and Albert R. Meyer. The complexity of the finite containment problem for petri nets. J. ACM, 28(3):561576, 1981.

[Sch87] Alexander Schrijver. Theory of Linear and Integer Programming. John Wiley and Sons, New York, 1987.

[ST77] George S. Sacerdote and Richard L. Tenney. The decidability of the reachability problem for vector addition systems (preliminary version). In Conference Record of the Ninth Annual ACM Symposium on Theory of Computing, 2-4 May 1977, Boulder, Colorado, USA, pages 61-76. ACM, 1977. 